\title{
SE DEBE EVITAR LA SOLDADURA DE BARRAS DE REFUERZO EN ESTRUCTURAS DE CONCRETO REFORZADO EN ZONAS SISMICAS DE MEXICO
}

\author{
Mario E. Rodríguez ${ }^{(1)}$, Javier Rodríguez Asabay ${ }^{(2)}$
}

\begin{abstract}
RESUMEN
Se estudia el efecto de la soldadura en las propiedades mecánicas de barras de refuerzo producidas de acuerdo con la normativa NMX-C-407 (ASTM A615). Se ensayaron a tensión a la falla en una máquina Universal un total de 54 muestras de diámetros de 1"(25.4 mm), 1-1/4" (31.8 mm) y 11/2” (38.1 mm). Las variables consideradas fueron: tipos de electrodo y de bisel, y el efecto del precalentamiento. La soldadura de las barras se llevó a cabo siguiendo procedimientos usuales de campo, sin precalentamiento de la barra, sin resecado de los electrodos, sin esmerilar las superficies a unir, etc., así como los establecidos por normativas específicas que aquí se describen. Con los resultados de los ensayes de las barras soldadas y de los valores obtenidos en las curvas esfuerzodeformación para estas barras se evalúan sus modos de falla y los principales parámetros que definen estas curvas. Los resultados de este estudio indican que no se deben soldar barras de refuerzo en estructuras en zonas sísmicas de México. Además, por las buenas características de soldabilidad de barras de refuerzo con aceros de baja aleación, especificados por la normativa Mexicana NMX-B-457 (ASTM A706), se recomienda empezar la producción en México de este tipo de refuerzo para ser empleado en estructuras en zonas sísmicas de nuestro país.
\end{abstract}

\begin{abstract}
This paper studies the effects of welding on mechanical properties of Mexican reinforcing bars that are produced following the NMX C-407 specification (ASTM 615). The reinforcing bars were welded using direct butt joints and arc welding. A total of 54 reinforcing bars were welded, with the following diameters, 1”(25.4 mm), 1-1/4” (31.8 mm) y 1-1/2” (38.1 mm), and were tested under tensile forces up to failure in an Universal Testing Machine. The parameters studied in this research were type of electrode, type of direct butt joint, and the effect of preheat on a reinforcing bar. Different welding procedures were followed for this study, namely, with and without bar preheating, without electrode preheating and without preparation of the base metal, as well as following welding procedures prescribed by current welding specifications described in this paper. Results obtained from the experimental research program conducted in this study were mainly stress-strain relationships for the welded rebars and types of failure modes. These results indicate that reinforcing bars currently produced in Mexico should not be welded in seismic areas of
\end{abstract}

\footnotetext{
Artículo recibido el 4 de julio de 2006 y aprobado para su publicación el 16 de febrero de 2007. Se aceptarán comentarios y/o discusiones hasta cinco meses después de su publicación

${ }^{1}$ Instituto de Ingeniería, UNAM, Ciudad Universitaria, Apartado Postal 70-290, Coyoacán, CP 04510, México,D.F, mrod@servidor.unam.mx

${ }^{2}$ Instituto de Ingeniería, UNAM, Ciudad Universitaria, Apartado Postal 70-472, Coyoacán, CP 04510, México,D.F, jasabay@yahoo.com.mx
} 
Mexico. Furthermore, due to the good weldability of low-alloy steel reinforcing bars (NMX-B-457 or ASTM A706), it is suggested to start in Mexico the production of these bars.

\section{INTRODUCCION}

En la industria de la construcción es práctica común que para lograr continuidad en barras de acero para refuerzo de concreto se tenga que recurrir a técnicas como el traslape. Esta solución se debe a que tanto los procesos constructivos como las dimensiones de los elementos estructurales obligan a traslapar las barras de refuerzo. Cuando el traslape no es una solución práctica, o no lo permitan los reglamentos de construcción, se recurre a técnicas como los conectores mecánicos y la soldadura con arco eléctrico con electrodo. Este tipo de solución se emplea principalmente en nuestro país en barras de diámetros grandes, mayores que $25 \mathrm{~mm}$; sin embargo, también existen casos en los que se sueldan o conectan varillas de $25 \mathrm{~mm}$ de diámetro. Un ejemplo de la mencionada restricción de reglamentos de construcción son las especificaciones para traslape del ACI 318-05 (ACI-318, 2005), las cuales no permiten traslapar barras de diámetro $38 \mathrm{~mm}$ o mayores.

En una gran mayoría de estructuras de concreto reforzado en México se emplea el acero de refuerzo que especifica la normativa mexicana NMX-C-407-ONNCCE-2001 (Norma Oficial Mexicana, 2001), la cual prácticamente sigue las especificaciones ASTM A615 (ASTM, 1992). En nuestro país, en la mayor parte de los casos se suelda con arco eléctrico, sin tomar en cuenta las recomendaciones de las normas existentes al respecto, como el precalentamiento de la barra, el esmerilado de las superficies a unir, el resecado de los electrodos, etc. Es necesario tomar en cuenta este tipo de recomendaciones, particularmente porque la norma mexicana NMX-C-407ONNCCE-2001 no establece un límite del contenido de carbono en la composición química de la barra de refuerzo. Se debe mencionar que el carbono es el responsable de los cambios en la microestructura en la zona afectada por el calor y de la dificultad para la operación de soldadura. Como se muestra en este trabajo, el contenido de carbono de las barras de refuerzo producidas en México es alto, lo que lleva a características pobres de soldabilidad. Un problema adicional que incide en la seguridad estructural de elementos de concreto reforzado en los que se emplean barras soldadas es la poca preparación de los soldadores y la deficiente supervisión del proceso, el cual se realiza generalmente en el lugar de la obra.

Si se considera que la seguridad de las construcciones en las cuales se utiliza barras de refuerzo soldadas no sólo depende de la resistencia de las barras, sino también de las uniones soldadas, se infiere que es relevante conocer las propiedades mecánicas de estas uniones. En este trabajo se describe un estudio experimental llevado a cabo en México con el objetivo de evaluar las propiedades mecánicas de barras de refuerzo soldadas, estas barras fueron producidas en nuestro país de acuerdo con la normativa mexicana NMX-C-407-ONNCCE-2001. En este estudio se ensayaron a tensión barras de refuerzo que fueron soldadas a tope directo por arco eléctrico con electrodo protegido. Se toma en cuenta principalmente tres variables: a) El tipo de electrodo empleado, b) El tipo de bisel con el que se forma la soldadura, y c) Empleo o no de un proceso de precalentamiento requerido por la normativa existente. 
Para la realización de este estudio se efectuaron ensayes en tensión a la falla de barras soldadas de acero de refuerzo de diámetros 1” (25.4 mm), 1-1/4” (31.8 mm) y 1-1/2” (38.1 mm) con números de designación 8,10 y 12, respectivamente. Con base en el análisis de los resultados obtenidos en esta investigación se evalúa el efecto de las tres variables anteriormente mencionadas en parámetros importantes que definen las propiedades mecánicas de barras soldadas, como son deformación última, esfuerzo último y esfuerzo de fluencia. Con base en el análisis de estos resultados se dan recomendaciones que sugieren evitar el empleo de barras soldadas para estructuras de concreto reforzado en zonas sísmicas de nuestro país.

\section{ASPECTOS RELEVANTES A CONSIDERAR EN LA SOLDADURA DE BARRAS DE REFUERZO}

Existen normas internacionales y nacionales para la soldadura del acero de refuerzo, como "El Reglamento de Soldadura Estructural Acero de Refuerzo ANSI/AWS D1.4-98” (AWS, 1998) y el "Procedimiento de soldadura Estructural Acero de Refuerzo", NMX-H-121-1988 (Norma Oficial Mexicana, 1988) que dan criterios para soldar barras de refuerzo de acero del tipo ASTM A615 grado 60 (ASTM, 1992). Sin embargo, la soldadura del acero de refuerzo en México se realiza, en la mayor parte de los casos, sin tomar en cuenta las recomendaciones especificadas por dichas normas. En general, el proceso de soldadura de barras de refuerzo se realiza en el lugar donde se desarrolla la obra, con una supervisión deficiente para el proceso y una baja calificación de los soldadores. En la mayoría de los casos no se precalientan las barras y en las superficies a unir no se eliminan las imperfecciones después de ser cortadas con oxicorte, además el ángulo de unión es inadecuado, el secado o el tipo de los electrodos no es el especificado, etc. La figura 1 muestra un proceso típico de soldadura de barras de refuerzo con arco eléctrico con electrodo protegido.

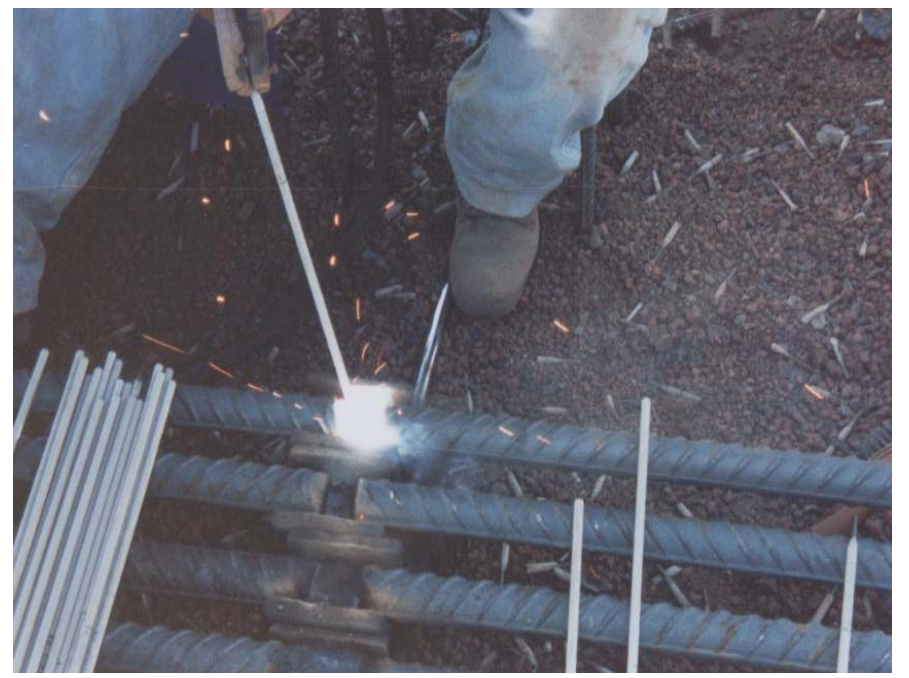

Figura 1. Soldadura de varillas

El arco eléctrico genera una gran cantidad de calor para fundir el electrodo, este calor induce transformaciones en la microestructura de la barra de refuerzo creando una región conocida como “Zona Afectada por el Calor” (ZAC). Las características de esta región dependen 
del contenido de carbono de las barras de refuerzo, del proceso y del procedimiento de soldadura utilizados. La figura 2 muestra una barra cortada después de haber sido soldada en esta investigación, con el objeto de mostrar la zona de la barra afectada por el calor en el proceso de soldadura. En esta investigación se observó que en la mayor parte de los ensayes en esta zona se produjo la fractura de la barra de manera frágil.

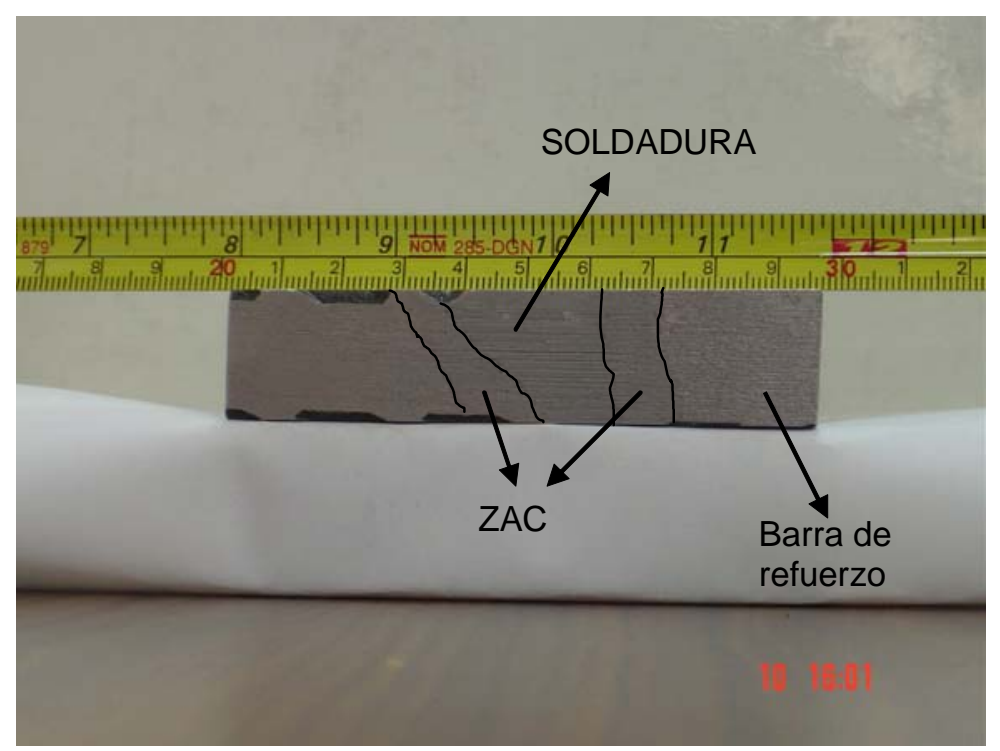

Figura 2. Zona Afectada por el Calor (ZAC) en una varilla soldada en esta investigación

Es necesario el precalentamiento de las varillas antes de soldarlas para reducir la velocidad de enfriamiento, evitar la contracción y la formación de una estructura llamada martensita, la cual confiere al acero una gran dureza, afectando de manera negativa otras propiedades mecánicas como la ductilidad (AWS, 1968). Las especificaciones de la ANSI/AWS D1.4-98 (AWS, 1998) recomienda el precalentamiento de las barras de refuerzo del tipo ASTM A615 antes de ser soldadas, de acuerdo con el valor del carbono equivalente, el cual se evalúa con un procedimiento que se describe posteriormente. El esmerilado de las superficies a unir, cortadas con oxígeno-acetileno, garantiza una mejora en la unión entre el metal base y la soldadura.

El calentamiento de los electrodos contribuye a minimizar el agrietamiento de la soldadura por la acción del hidrógeno. El Reglamento ANSI/AWS D1.4 (AWS, 1998), señala que los electrodos de la serie E90XX-X, los cuales son recomendados para soldar el acero de refuerzo del tipo ASTM A615, deberán ser calentados a una temperatura no menor de $370^{\circ} \mathrm{C}$ durante por lo menos 2 horas, si los electrodos han estado expuestos a la atmósfera por mas de 5 horas. Sin embargo, es común que en nuestro país esta recomendación se siga de manera inadecuada, ya que se utilizan lámparas sin control de temperatura.

Por su contenido de carbono los aceros se clasifican en aceros de bajo carbono, que son los que tienen hasta $0.30 \%$ de carbono, medio carbono, desde $0.30 \%$ hasta $0.60 \%$ de carbono y alto carbono con mas de $0.60 \%$ de carbono (ASM, 1990). Como se describe en lo que sigue, el 
contenido de carbono es relevante para lograr que la región soldada sea más resistente que el metal base. En los aceros con menos del 0.25\% de carbono, la condición anterior se logra por el bajo contenido de carbono y en general el calentamiento no provoca cambios importantes en el metal base cercano a la región soldada. Durante el proceso de soldadura, el metal base adyacente a la soldadura se calienta por encima de la temperatura crítica de $727^{\circ} \mathrm{C}$, provocando cambios en la microestructura del acero, que al enfriarse puede llevar a la creación de nuevas estructuras con propiedades mecánicas diferentes del metal base. Como los aceros de bajo carbono tienen una baja templabilidad (facilidad con la que un acero puede aumentar su dureza) rara vez aumentan su dureza en el proceso de soldadura. Los problemas de poca soldabilidad generalmente aparecen en los aceros con un contenido de carbono mayor que $0.45 \%$, ya que en este caso se requiere generalmente un precalentamiento o un postcalentado para reducir los efectos provocados por el calor en las propiedades mecánicas del acero.

Otro aspecto importante que influye en la calidad de barras de refuerzo soldadas es que la norma NMX-C-407-ONNCCE-2001, con la cual se fabrican las barras empleadas en nuestro país, no estipula límites de contenido de carbono en este tipo de barras, lo que llevaría a tener en nuestro país barras de refuerzo de los tipos medio o alto carbono anteriormente descritos, con lo cual estas barras tendrían limitaciones importantes para lograr una calidad adecuada de soldadura. Por el contrario, la normativa mexicana NMX-B-457 (Norma Oficial Mexicana, 1988) para aceros de refuerzo de baja aleación (los cuales aún no se producen en nuestro país), la cual es equivalente a la normativa ASTM A706 (ASTM, 1992), especifica que las barras que se producen cumpliendo esta normativa son de calidad soldable. Para este fin, esta normativa especifica límites máximos para el contenido de carbono y para el carbono equivalente, con valores de $0.30 \%$ y $0.55 \%$, respectivamente. Este tipo de acero de refuerzo de baja aleación tiene un esfuerzo de fluencia mínimo especificado igual al de la normativa NMX-C-407-ONNCCE2001, es decir de 4,200 kg/cm2; con resistencia mínima a la tensión de 1.25 veces el esfuerzo de fluencia medido. Es de interés que la normativa ASTM A 706 no es reciente, fue introducida en 1974 en los Estados Unidos (Gustafson y Felder, 1991). En una publicación reciente (Gustafson, 2007) se comentan diversas características de las barras de refuerzo que siguen la normativa ASTM A 706, tanto técnicas, como de costos y de disponibilidad en los Estados Unidos.

La capacidad de un material para ser soldado, que en este trabajo se denomina soldabilidad, depende de la composición química del acero, y se evalúa con el parámetro llamado Carbono Equivalente (CE). Este parámetro se define con expresiones empíricas. Por ejemplo, el Reglamento de Soldadura para aceros de refuerzo ANSI/AWS D1.4-98 (AWS, 1998) emplea la siguiente expresión para el cálculo del parámetro CE:

$$
\text { C.E. }=\% C+\frac{\% M n}{6}
$$

El Procedimiento de Soldadura Estructural Acero de Refuerzo de la NMX-H-121-1988 (Norma Oficial Mexicana, 1988) emplea la siguiente expresión para evaluar CE:

$$
\text { C.E. }=\% C+\frac{\% M n}{6}+\frac{\% C u}{40}+\frac{\% N i}{20}+\frac{\% C r}{10}-\frac{\% M o}{50}-\frac{\% V}{10}
$$


Las expresiones anteriores sugieren que la soldabilidad de los aceros al carbono depende principalmente del contenido de carbono. Para los aceros de bajo carbono la soldabilidad es excelente, es buena y regular para los aceros de medio carbono y pobre para los aceros de alto carbono (AWS, 1987). Los criterios para esta clasificación se comentan en lo que sigue.

Los aceros con un máximo de $0.30 \%$ de contenido de carbono son adecuados para soldar, en general se pueden soldar logrando un comportamiento de la barra del tipo dúctil y por ejemplo son los más usados por los herreros para hacer ventanas, puertas, etc. Los aceros de medio y alto carbono son más difíciles de soldar ya que con bastante facilidad se puede formar martensita en la zona afectada por el calor, causando por lo tanto, una soldadura del tipo frágil. La tabla 1 muestra las características de soldabilidad de algunos aceros de acuerdo con el contenido de carbono y CE.

Como el parámetro CE esta íntimamente ligado con el contenido de carbono en los aceros, se suele utilizar el diagrama carbono-carbono equivalente para localizar los aceros de acuerdo a su soldabilidad, que se describe a continuación. Las coordenadas del diagrama son el contenido de carbono en la escala vertical y el carbono equivalente en la escala horizontal. En el diagrama se delimitan tres zonas. La zona I comprende a los aceros de soldabilidad óptima, los que son aceros con contenidos de carbono sumamente bajos. La zona II abarca aceros de buena a regular soldabilidad, como por ejemplo el acero ASTM 36, el cual es muy empleado para la construcción de estructuras de acero en nuestro país, y el acero ASTM 706. La zona III comprende a los aceros con alto riesgo de falla en el caso de ser soldados. Las zonas que aquí se describen se muestran en la figura 3. En esta figura se emplean los datos de la composición química de las barras de refuerzo utilizadas en esta investigación (indicadas como Aceros ASTM A615 en la figura), los que fueron proporcionados por el fabricante a través de un certificado de calidad expedido por un laboratorio.

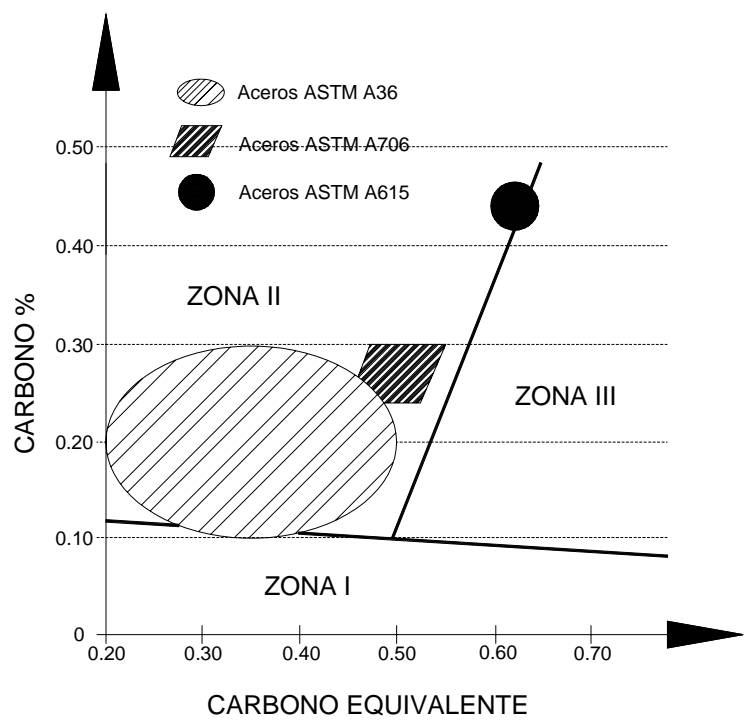

Figura 3. Diagrama del contenido de carbono contra el carbono equivalente 
Como se aprecia en la figura 3, dependiendo del contenido de carbono y el valor del CE, las varillas de refuerzo producidas en nuestro país de acuerdo con la normativa NMX-C-407ONNCCE-2001 (equivalentes a las normas ASTM A615) pueden tener niveles de soldabilidad de regular a pobre, por lo que las normativas existentes para soldar este tipo de barras especifican el precalentamiento de las barras, así como electrodos de bajo contenido de hidrógeno. En esta investigación se evalúan si estas especificaciones ayudan a mejorar el problema de la pobre soldabilidad de las barras de refuerzo producidas en nuestro país.

Tabla 1. Soldabilidad de algunos aceros con base en el contenido de carbono y CE

\begin{tabular}{|c|c|c|c|}
\hline Acero & \% de Carbono & $\begin{array}{c}\text { Carbono } \\
\text { Equivalente }\end{array}$ & $\begin{array}{c}\text { Niveles de } \\
\text { soldabilidad }^{\mathrm{d}}\end{array}$ \\
\hline ASTM A36 & 0.10 a $0.30^{\mathrm{a}}$ & 0.20 a $0.50^{\mathrm{a}}$ & Buena $^{\mathrm{b}}$ \\
\hline ASTM A615 Grado 60 & 0.417 a $0.443^{\mathrm{b}}$ & 0.59 a $0.62^{\mathrm{b}}$ & Regular \\
\hline ASTM A706 Grado 60 & Máx 0.30 & ${\text { Máx } 0.55^{\mathrm{C}}}^{\mathrm{c}}$ & Buena \\
\hline
\end{tabular}

Notas:

a.- ASM, 1997

b.- Composición química de las varillas estudiadas en esta investigación obtenidas del fabricante y CE calculado para estas varillas

c.- ASTM A706, 1992

d.- AWS, 1987

\section{DESCRIPCION DE LA INVESTIGACION EXPERIMENTAL}

\section{Metodología y materiales}

En esta investigación se soldaron y ensayaron a tensión un total de 54 muestras de barras de refuerzo clasificadas como $\mathrm{N}^{0} 8,10$ y 12. Las barras fueron soldadas en laboratorio por un soldador calificado, empleando la técnica de tope directo. Los ensayes se llevaron a la falla en una máquina tipo Universal de 200 ton de capacidad. Además, con fines de comparación se ensayaron 3 barras sin soldadura para cada uno de los diámetros estudiados. Las muestras ensayadas tuvieron una longitud de $90 \mathrm{~cm}$ con una longitud mínima de $30 \mathrm{~cm}$ entre mordazas de la máquina Universal.

De los ensayes a tensión se obtuvieron curvas esfuerzo-deformación hasta la ruptura de las barras. Para el registro de las deformaciones en los ensayes efectuados se utilizó un dispositivo construido en el Instituto de Ingeniería para una investigación anterior (Rodríguez y Botero, 1996). La figura 4 muestra este dispositivo en una barra de refuerzo que ha llegado a la falla en un ensaye para este estudio. El dispositivo cuenta con un conjunto de strain gauges de puente completo adheridos a una lámina delgada de acero que al flexionarse provoca un cambio en su resistencia eléctrica, la cual es registrada por los strain gauges. La señal registrada es amplificada y registrada en un sistema de adquisición de datos para ser capturada en una computadora personal donde previamente se configura para obtener una medida final en 
milímetros. Se debe mencionar que los laboratorios nacionales de control de calidad no cuentan con este tipo de dispositivos para medir deformaciones en las barras que se ensayan a tensión, por lo que el control de calidad en laboratorio de estas barras se limita principalmente a evaluar esfuerzos de fluencia y de ruptura.

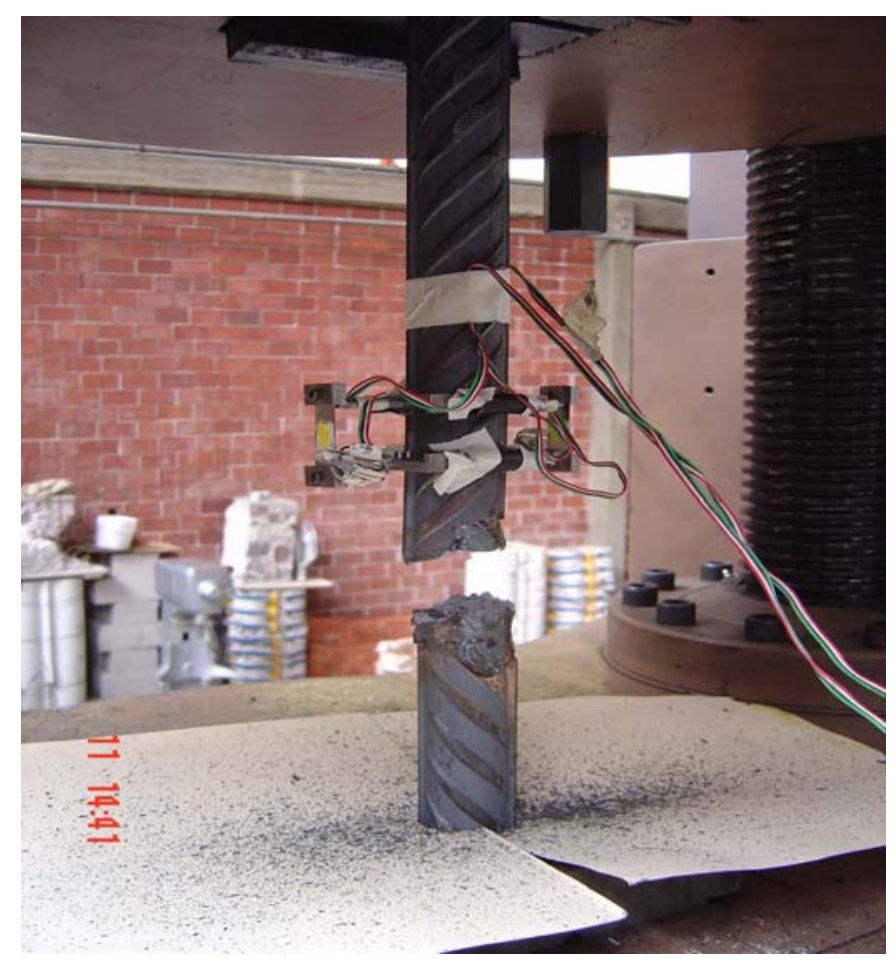

Figura 4. Dispositivo para medir deformaciones

Se utilizaron electrodos E-7018 y E-9018-B3 de diámetros 1/8” y 5/32”. Además, se empleó una máquina de soldar de corriente directa marca "Haggluns". El corte y precalentamiento se llevó a cabo con equipo de oxígeno-acetileno y un gis térmico para medir la temperatura de precalentamiento requerida.

Las tablas 2 a la 4 muestran la composición química de las varillas $\mathrm{N}^{\mathrm{o}}$ 8, 10 y 12, respectivamente, que se emplearon en este estudio. Esta información fue proporcionada por el fabricante.

Tabla 2. Composición química de barras $\mathbf{N}^{0} 8$.

\begin{tabular}{|c|c|c|c|c|c|c|c|}
\hline \% C & \% Si & \% Mn & \% P & \% S & \% Cr & \% Ni & \% Cu \\
\hline 0.417 & 0.156 & 1.011 & 0.013 & 0.024 & 0.139 & 0.084 & 0.289 \\
\hline
\end{tabular}


Tabla 3. Composición química de barras $\mathbf{N}^{0} 10$.

\begin{tabular}{|c|c|c|c|c|c|c|c|}
\hline \% C & \% Si & \% Mn & \% P & \% S & \% Cr & \% Ni & $\% \mathrm{Cu}$ \\
\hline 0.443 & 0.207 & 1.029 & 0.021 & 0.033 & 0.132 & 0.102 & 0.491 \\
\hline
\end{tabular}

Tabla 4. Composición química de barras $\mathbf{N}^{0} 12$.

\begin{tabular}{|l|l|l|l|l|l|l|l|}
\hline \% C & \% Si & \% Mn & \% P & \% S & \% Cr & \% Ni & \% Cu \\
\hline 0.441 & 0.156 & 1.060 & 0.017 & 0.029 & 0.088 & 0.097 & 0.444 \\
\hline
\end{tabular}

Con base en la información de las tablas 2 a la 4 se calculó el parámetro CE (carbono equivalente) con la ecuación (1) (ANSI/AWS D1.4-98) y con la ecuación (2) (NMX-H-121). Los resultados de emplear estas ecuaciones se muestran en la tabla 5. Considerando la relación entre CE y la soldabilidad en barras de refuerzo con características de la normativa NMX-C-407, los resultados de la tabla 5 indican que las barras de refuerzo que actualmente se producen en México tendrían en general un nivel pobre de soldabilidad.

Tabla 5. Carbono equivalente de las varillas $N^{\circ} 8,10$ y 12

\begin{tabular}{|c|c|c|}
\hline Número de varilla & $\begin{array}{c}\text { C. E. } \\
\text { Ec. (1) }\end{array}$ & $\begin{array}{c}\text { C. E. } \\
\text { Ec. (2) }\end{array}$ \\
\hline $\mathrm{N}^{\mathrm{o}} 8$ & 0.59 & 0.61 \\
\hline $\mathrm{N}^{\mathrm{o}} 10$ & 0.61 & 0.65 \\
\hline $\mathrm{N}^{\mathrm{o}} 12$ & 0.62 & 0.64 \\
\hline
\end{tabular}

Con los resultados del carbono equivalente y empleando la tabla 5.2 de ANSI/AWS D1.498(AWS, 1998) se obtiene que para soldar las barras de refuerzo empleadas en este estudio es necesario una temperatura de precalentamiento de $150^{\circ} \mathrm{C}$.

\section{Clasificación de las barras soldadas ensayadas}

La tabla 6 muestra las características y número de las barras de refuerzo soldadas empleadas en este estudio. Como se aprecia en la referida tabla, se consideraron seis grupos de barras soldadas, identificadas en la columna (1) de la tabla con las letras A a la F. La columna (2) de la tabla indica el número de identificación de la barra soldada. La información de las columnas (3) y (4) indica si las barras se soldaron sin o con precalentamiento, respectivamente. La columna (5) indica que las barras se soldaron con bisel B1, correspondiente a varillas soldadas en posición vertical, figura 5. La columna (6) indica que las barras se soldaron con bisel B2, correspondiente a varillas soldadas en posición horizontal, figura 6. Las columnas (7) y (8) indican el tipo de electrodo empleado en el estudio experimental, E7018 y E9018 respectivamente. La columna (9) indica el total de muestras por diámetro. 
Tabla 6 Características y número de barras de refuerzo soldadas y ensayadas

\begin{tabular}{|c|c|c|c|c|c|c|c|c|}
\hline Grupo & $\begin{array}{l}\mathrm{N}^{0} \mathrm{De} \\
\text { Varilla }\end{array}$ & $\begin{array}{c}\text { Sin pre- } \\
\text { calentamiento }\end{array}$ & $\begin{array}{c}\text { Con pre- } \\
\text { calentamiento }\end{array}$ & B1 & B2 & E-70 & E-90 & Total \\
\hline (1) & (2) & (3) & (4) & (5) & (6) & (7) & (8) & (9) \\
\hline \multirow{3}{*}{ A } & 8 & $\mathrm{X}$ & & $\mathrm{X}$ & & $\mathrm{X}$ & & 3 \\
\hline & 10 & $\mathrm{X}$ & & $\mathrm{X}$ & & $\mathrm{X}$ & & 3 \\
\hline & 12 & $\mathrm{X}$ & & $\mathrm{X}$ & & $\mathrm{X}$ & & 3 \\
\hline \multirow{3}{*}{ B } & 8 & $X$ & & $\mathrm{X}$ & & & $X$ & 3 \\
\hline & 10 & $X$ & & $X$ & & & $X$ & 3 \\
\hline & 12 & $X$ & & $\mathrm{X}$ & & & $X$ & 3 \\
\hline \multirow{3}{*}{$\mathbf{C}$} & 8 & & $X$ & $X$ & & $X$ & & 3 \\
\hline & 10 & & $X$ & $X$ & & $X$ & & 3 \\
\hline & 12 & & $X$ & $X$ & & $X$ & & 3 \\
\hline \multirow{3}{*}{ D } & 8 & & $X$ & $X$ & & & $\mathrm{X}$ & 3 \\
\hline & 10 & & $X$ & $\mathrm{X}$ & & & $X$ & 3 \\
\hline & 12 & & $X$ & $\mathrm{X}$ & & & $X$ & 3 \\
\hline \multirow{3}{*}{$\mathbf{E}$} & 8 & $X$ & & & $\mathrm{X}$ & & $X$ & 3 \\
\hline & 10 & $X$ & & & $X$ & & $X$ & 3 \\
\hline & 12 & $X$ & & & $X$ & & $X$ & 3 \\
\hline \multirow{3}{*}{$\mathbf{F}$} & 8 & & $\mathrm{X}$ & & $\mathrm{X}$ & & $\mathrm{X}$ & 3 \\
\hline & 10 & & $\mathrm{X}$ & & $\mathrm{X}$ & & $\mathrm{X}$ & 3 \\
\hline & 12 & & $X$ & & $X$ & & $X$ & 3 \\
\hline & & & & & & & Total & 54 \\
\hline
\end{tabular}

\section{RESULTADOS DEL ESTUDIO EXPERIMENTAL}

La tabla 7 muestra un resumen de resultados encontrados en este estudio en los ensaye a tensión de las barras de control sin soldadura. Las columnas (2) y (3) de esta tabla muestran la carga máxima $(\mathrm{P})$ y el esfuerzo máximo $\left(\mathrm{F}_{\mathrm{su}}\right)$, respectivamente, la columna (4) muestra el esfuerzo de fluencia $\left(\mathrm{F}_{\mathrm{y}}\right)$, la col (5) muestra el módulo de elasticidad (Es), la columna (6) muestra la deformación máxima $\left(\varepsilon_{\mathrm{su}}\right)$ y la columna (7) muestra la relación entre el esfuerzo máximo alcanzado y el esfuerzo de fluencia especificado de $4,200 \mathrm{~kg} / \mathrm{cm}^{2}$. 


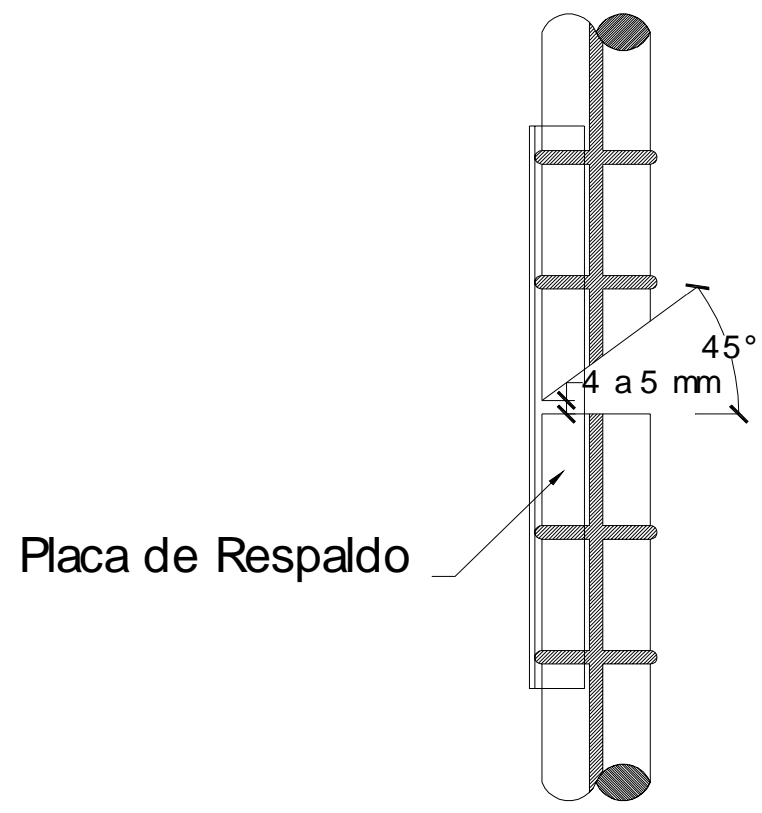

Figura 5. Bisel tipo B1 para varillas soldadas en posición vertical

\section{Placa de Respaldo}

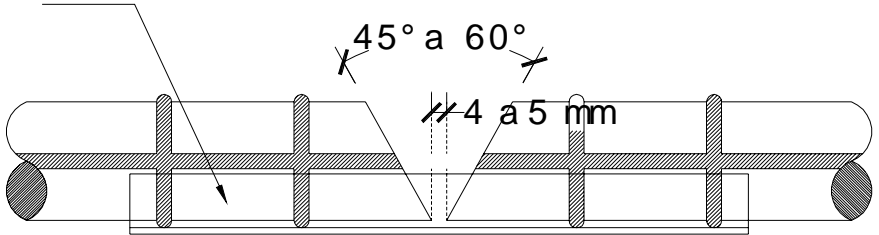

Figura 6. Bisel tipo B2 para varillas soldadas en posición horizontal

Tabla 7. Resultados del ensaye a tensión de barras sin soldadura

\begin{tabular}{|c|c|c|c|c|c|c|}
\hline $\begin{array}{c}\mathrm{N}^{\mathrm{o}} \text { de } \\
\text { barra }\end{array}$ & $\begin{array}{c}\mathrm{P} \\
(\mathrm{kg})\end{array}$ & $\begin{array}{c}\mathrm{F}_{\mathrm{su}} \\
\left(\mathrm{kg} / \mathrm{cm}^{2}\right)\end{array}$ & $\begin{array}{c}\mathrm{F}_{\mathrm{y}} \\
\left(\mathrm{kg} / \mathrm{cm}^{2}\right)\end{array}$ & $\begin{array}{c}\mathrm{E}_{\mathrm{s}} \\
\left(\mathrm{kg} / \mathrm{cm}^{2}\right)\end{array}$ & $\begin{array}{c}\varepsilon_{\mathrm{su}} \\
\%\end{array}$ & $\mathrm{~F}_{\mathrm{su}} / \mathrm{F}_{\mathrm{y}}$ \\
\hline$(1)$ & $(2)$ & $(3)$ & $(4)$ & $(5)$ & $(6)$ & $(7)$ \\
\hline 8 & 35580 & 7020 & 4320 & 1797140 & 12 & 1.67 \\
\hline 10 & 60720 & 7650 & 4370 & 2222990 & 10 & 1.82 \\
\hline 12 & 84645 & 7425 & 4370 & 2121550 & 11.7 & 1.76 \\
\hline
\end{tabular}


BARRAS TESTIGO

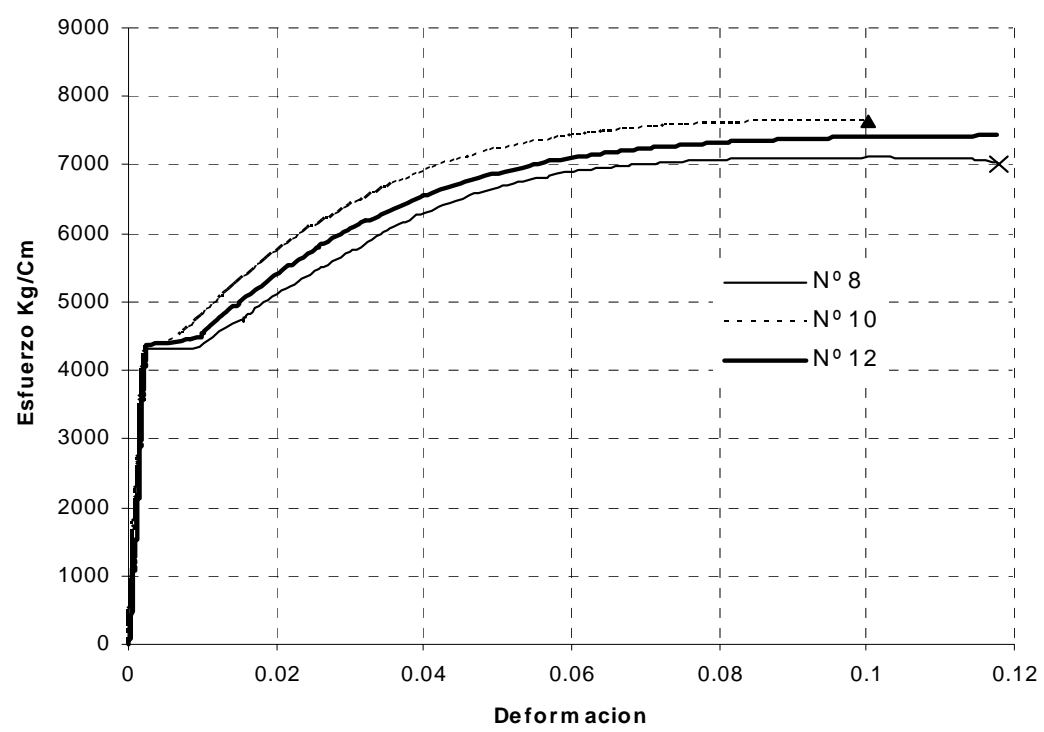

Figura. 7. Diagrama esfuerzo-deformación de barras sin soldadura

Como se ha mencionado, en esta investigación se estudia el efecto tres variables: la temperatura inicial del metal base, el tipo de bisel y el tipo de electrodo para las barras soldadas. Para este fin, los grupos definidos en la tabla 6 se reagrupan en siete casos, numerados del I al VII, de acuerdo con el efecto de estas variables. Se tienen 3 casos para el estudio del efecto de la temperatura inicial del metal base manteniendo fijos el bisel y el tipo de electrodo, los cuales se identifican como casos de I a III. Para el estudio del efecto del bisel se mantienen fijos la temperatura y el tipo de electrodo, y se tienen dos casos, identificados como IV y V. Para el estudio del efecto del electrodo, se mantienen fijos la temperatura y el bisel, y se tienen dos casos, identificados como VI y VII. De esta manera se tienen siete casos por cada uno de los diámetros considerados en este estudio, los que se resume a continuación.

a) Efecto de la temperatura inicial del metal base

Caso I- Grupos A y C (B1, E70, sin y con precalentamiento)

Caso II- Grupos B y D (B1, E90, sin y con precalentamiento)

Caso III- Grupos E y F (B2, E90, sin y con precalentamiento)

\section{b) Efecto del tipo de bisel}

Caso IV- Grupos B y E (E90, sin precalentamiento, B1 y B2)

Caso V- Grupos D y F (E90, con precalentamiento, B1 y B2)

\section{c) Efecto del tipo de electrodo}

Caso VI- Grupos A y B (B1, sin precalentamiento, E70 y E90)

Caso VII- Grupos C y D (B1, con precalentamiento, E70 y E90) 
Los resultados obtenidos de los ensaye a tensión de las barras soldadas se promediaron por cada muestra de tres barras respectivas y los resultados se resumen en las tablas 8 a la 10, correspondientes a las barras $\mathrm{N}^{\mathrm{o}}$ 8, 10 y 12, respectivamente. La columna (1) de estas tres tablas, se refiere a los seis grupos de barras soldadas descritos anteriormente. Las columnas (2) y (3) muestran la carga máxima $(\mathrm{P})$ y el esfuerzo máximo $\left(\mathrm{F}_{\mathrm{su}}\right)$, respectivamente, la columna (4) muestra el esfuerzo de fluencia $\left(\mathrm{F}_{\mathrm{y}}\right)$, la columna (5) muestra el módulo de elasticidad ( $\left.\mathrm{E}_{\mathrm{s}}\right)$, la columna (6) muestra la deformación máxima $\left(\varepsilon_{\mathrm{su}}\right)$ y la columna (7) muestra la relación entre el esfuerzo máximo y el esfuerzo de fluencia especificado de $4,200 \mathrm{~kg} / \mathrm{cm}^{2}$.

Tabla 8. Resultados promedio de ensayes a tensión de barras soldadas $\mathbf{N}^{\circ} 8$

\begin{tabular}{|c|c|c|c|c|c|c|}
\hline Grupos & $\begin{array}{c}\mathrm{P} \\
(\mathrm{kg})\end{array}$ & $\begin{array}{c}\mathrm{F}_{\mathrm{su}} \\
\left(\mathrm{kg} / \mathrm{cm}^{2}\right)\end{array}$ & $\begin{array}{c}\mathrm{F}_{\mathrm{y}} \\
\left(\mathrm{kg} / \mathrm{cm}^{2}\right)\end{array}$ & $\begin{array}{c}\mathrm{E}_{\mathrm{s}} \\
\left(\mathrm{kg} / \mathrm{cm}^{2}\right)\end{array}$ & $\begin{array}{c}\varepsilon_{\mathrm{su}} \\
\%\end{array}$ & $\mathrm{~F}_{\mathrm{su}} / \mathrm{F}_{\mathrm{y}}$ \\
\hline$(1)$ & $(2)$ & $(3)$ & $(4)$ & $(5)$ & $(6)$ & $(7)$ \\
\hline A & 23500 & 4635 & 4249 & 1991770 & 1.0 & 1.10 \\
\hline B & 28630 & 5647 & 4341 & 2014820 & 2.77 & 1.45 \\
\hline C & 23460 & 4627 & 4075 & 1668980 & 1.08 & 1.10 \\
\hline D & 31890 & 6290 & 4228 & 1720840 & 3.53 & 1.49 \\
\hline E & 35490 & 7000 & 4091 & 1656410 & 7.79 & 1.67 \\
\hline F & 33135 & 6536 & 4319 & 1608260 & 5.82 & 1.51 \\
\hline
\end{tabular}

Tabla 9. Resultados promedio de ensayes a tensión de barras soldadas $\mathbf{N}^{\mathbf{0}} \mathbf{1 0}$

\begin{tabular}{|c|c|c|c|c|c|c|}
\hline Grupos & $\begin{array}{c}\mathrm{P} \\
(\mathrm{kg})\end{array}$ & $\begin{array}{c}\mathrm{F}_{\mathrm{su}} \\
\left(\mathrm{kg} / \mathrm{cm}^{2}\right)\end{array}$ & $\begin{array}{c}\mathrm{F}_{\mathrm{y}} \\
\left(\mathrm{kg} / \mathrm{cm}^{2}\right)\end{array}$ & $\begin{array}{c}\mathrm{E}_{\mathrm{s}} \\
\left(\mathrm{kg} / \mathrm{cm}^{2}\right)\end{array}$ & $\begin{array}{c}\varepsilon_{\mathrm{su}} \\
\%\end{array}$ & $\mathrm{~F}_{\mathrm{su}} / \mathrm{F}_{\mathrm{y}}$ \\
\hline$(1)$ & $(2)$ & $(3)$ & $(4)$ & $(5)$ & $(6)$ & $(7)$ \\
\hline $\mathbf{A}$ & 30806 & 3880 & - & 1618810 & 0.5 & 0.92 \\
\hline B & 41737 & 5257 & 4285 & 1733970 & 1.93 & 1.25 \\
\hline $\mathbf{C}$ & 33072 & 4165 & 4280 & 2014170 & 0.73 & 0.99 \\
\hline $\mathbf{D}$ & 41808 & 5266 & 4310 & 1859990 & 1.16 & 1.25 \\
\hline $\mathbf{E}$ & 46902 & 5907 & 4380 & 2170290 & 1.59 & 1.41 \\
\hline F & 52194 & 6585 & 4370 & 2331240 & 2.90 & 1.57 \\
\hline
\end{tabular}

La información completa para un estudio comparativo del efecto de las variables estudiadas empleando las curvas experimentales esfuerzo-deformación obtenidas en esta investigación para cada una de las 54 barras soldadas ensayadas se puede encontrar en el reporte de Rodríguez y Rodríguez (2007). En este trabajo se presenta un resumen de estos resultados. En las figuras 8 a la 16 se muestran las curvas promedio esfuerzo-deformación para las barras de diámetros No 8, 10 y 12, para los seis grupos de barras estudiadas (grupos A al F), agrupados de manera de evaluar de manera sencilla el efecto de precalentamiento, es decir los casos I, II y III. La leyenda XT en las figuras 8 a 16 significa sin precalentamiento y T con precalentamiento. 
Tabla 10. Resultados promedio de ensayes a tensión de barras soldadas № 12

\begin{tabular}{|c|c|c|c|c|c|c|}
\hline Grupos & $\begin{array}{c}\mathrm{P} \\
(\mathrm{kg})\end{array}$ & $\begin{array}{c}\mathrm{F}_{\mathrm{su}} \\
\left(\mathrm{kg} / \mathrm{cm}^{2}\right)\end{array}$ & $\begin{array}{c}\mathrm{F}_{\mathrm{y}} \\
\left(\mathrm{kg} / \mathrm{cm}^{2}\right)\end{array}$ & $\begin{array}{c}\mathrm{E}_{\mathrm{s}} \\
\left(\mathrm{kg} / \mathrm{cm}^{2}\right)\end{array}$ & $\begin{array}{c}\varepsilon_{\mathrm{su}} \\
\%\end{array}$ & $\mathrm{~F}_{\mathrm{su}} / \mathrm{F}_{\mathrm{y}}$ \\
\hline$(1)$ & $(2)$ & $(3)$ & $(4)$ & $(5)$ & $(6)$ & $(7)$ \\
\hline A & 41699 & 3658 & 3880 & 2119260 & 0.43 & 0.87 \\
\hline B & 62156 & 5452 & 4220 & 2146250 & 1.80 & 1.30 \\
\hline C & 53197 & 4666 & 4050 & 1514650 & 1.59 & 1.11 \\
\hline D & 58579 & 5139 & 4340 & 2598840 & 1.00 & 1.22 \\
\hline E & 62771 & 5487 & 3995 & 1696000 & 2.30 & 1.31 \\
\hline F & 71100 & 6237 & 4090 & 1251940 & 4.90 & 1.48 \\
\hline
\end{tabular}

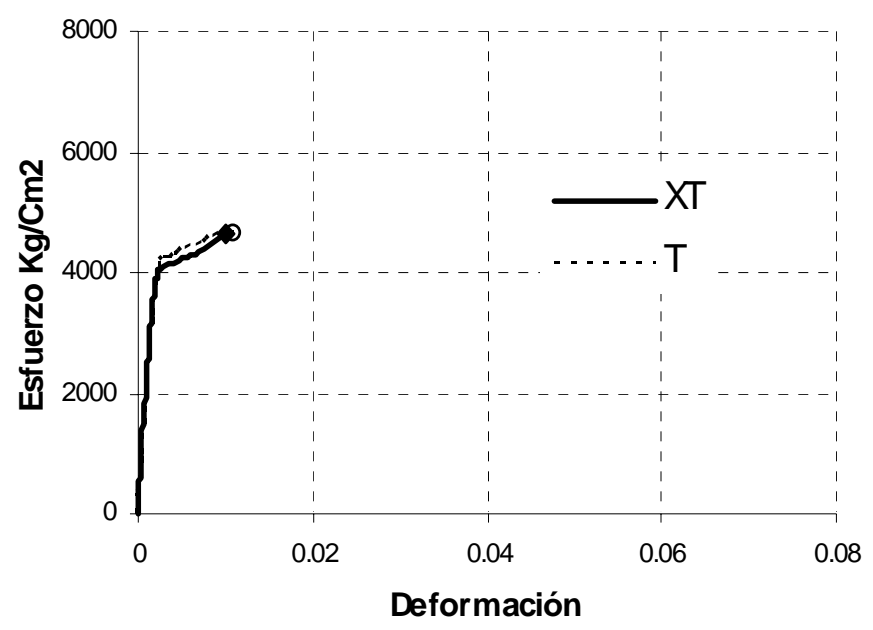

Figura. 8 Diagrama esfuerzo-deformación promedio de barras soldadas No 8 . Caso I

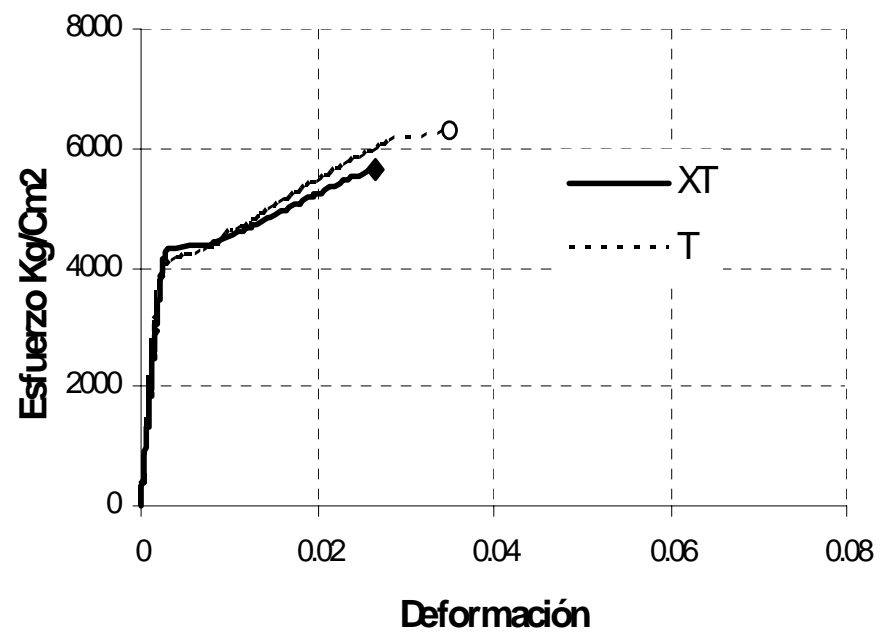

Figura. 9 Diagrama esfuerzo-deformación promedio de barras soldadas No 8. Caso II 


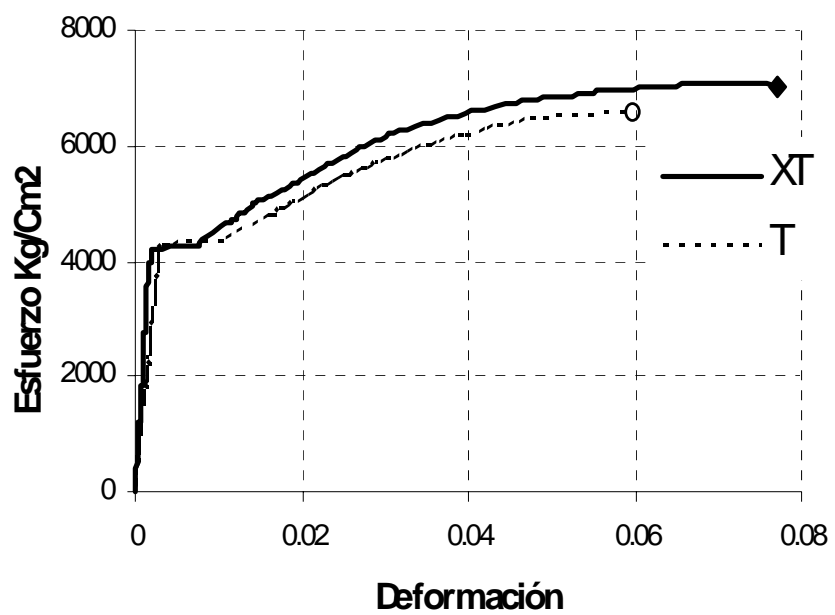

Figura. 10 Diagrama esfuerzo-deformación promedio de barras soldadas No 8. Caso III

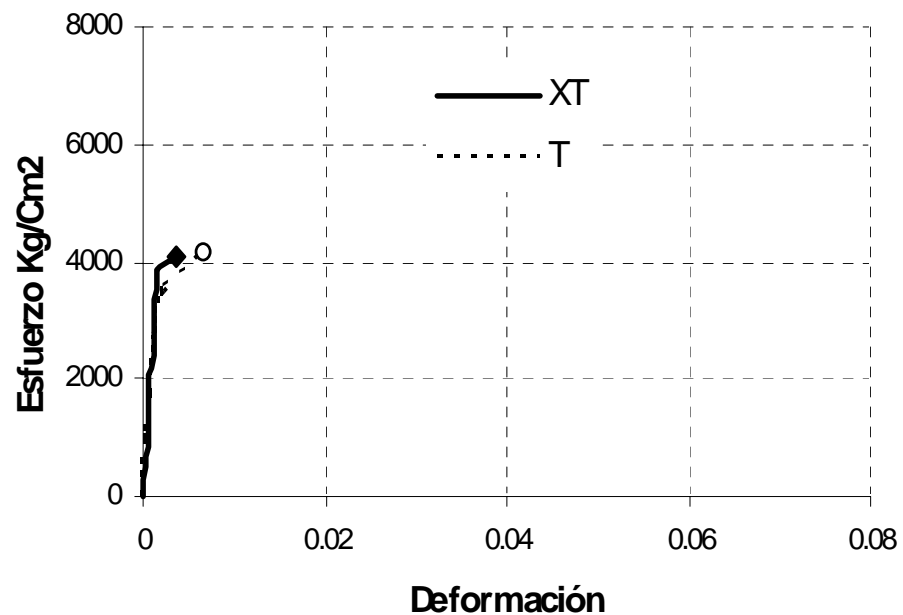

Figura. 11 Diagrama esfuerzo-deformación promedio de barras soldadas No 10. Caso I

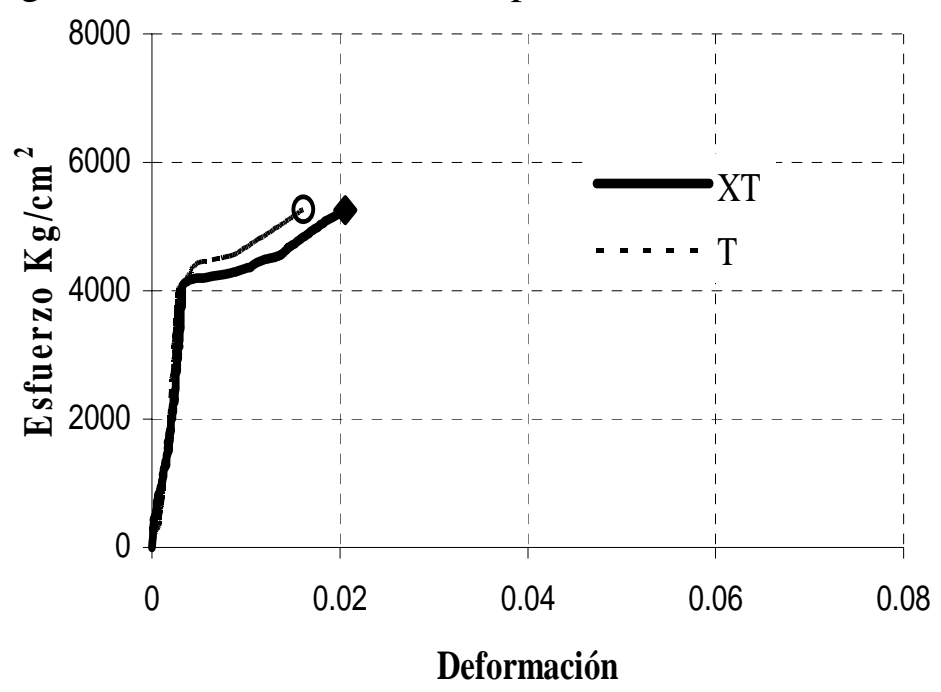

Figura. 12 Diagrama esfuerzo-deformación promedio de barras soldadas No 10. Caso II 


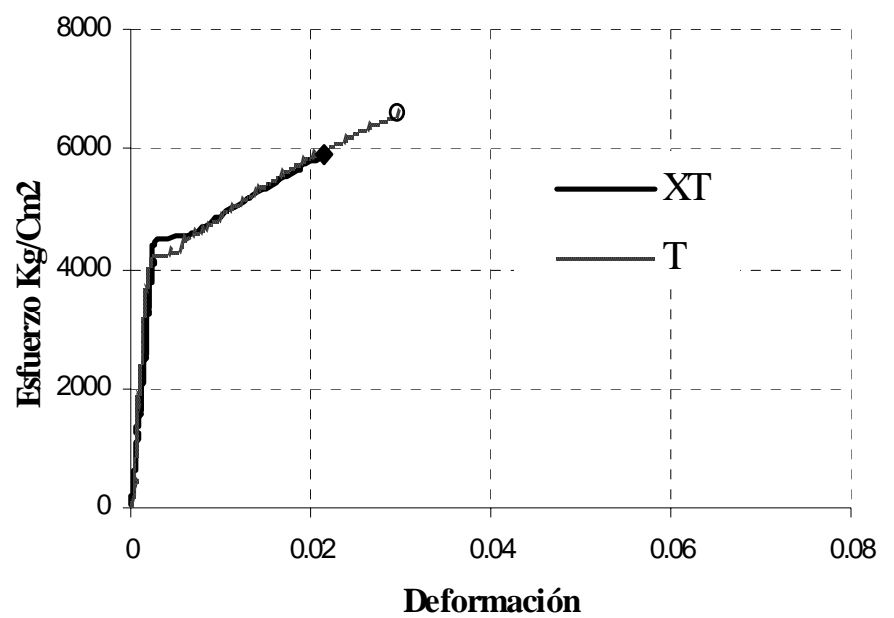

Figura. 13 Diagrama esfuerzo-deformación promedio de barras soldadas No 10. Caso III

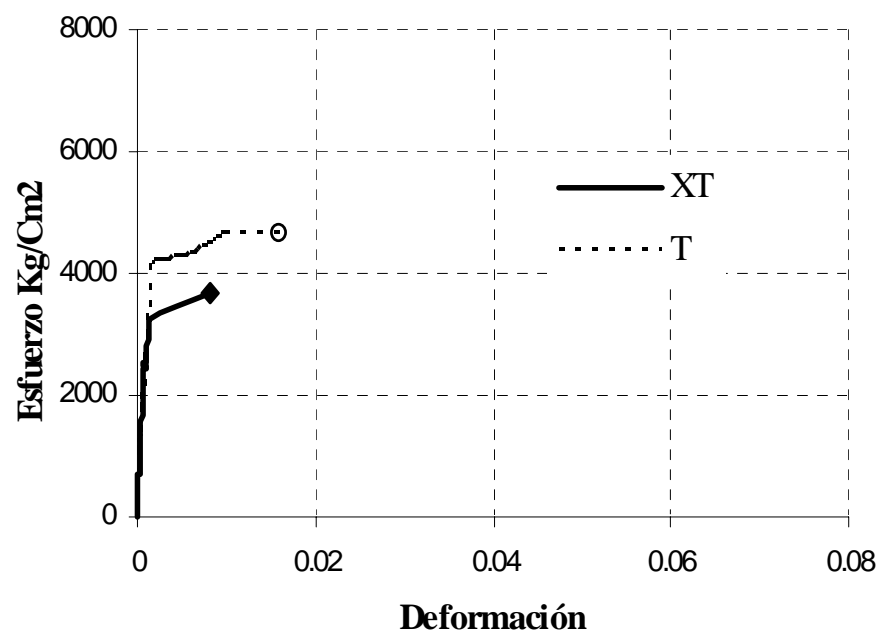

Figura. 14 Diagrama esfuerzo-deformación promedio de barras soldadas No 12. Caso I

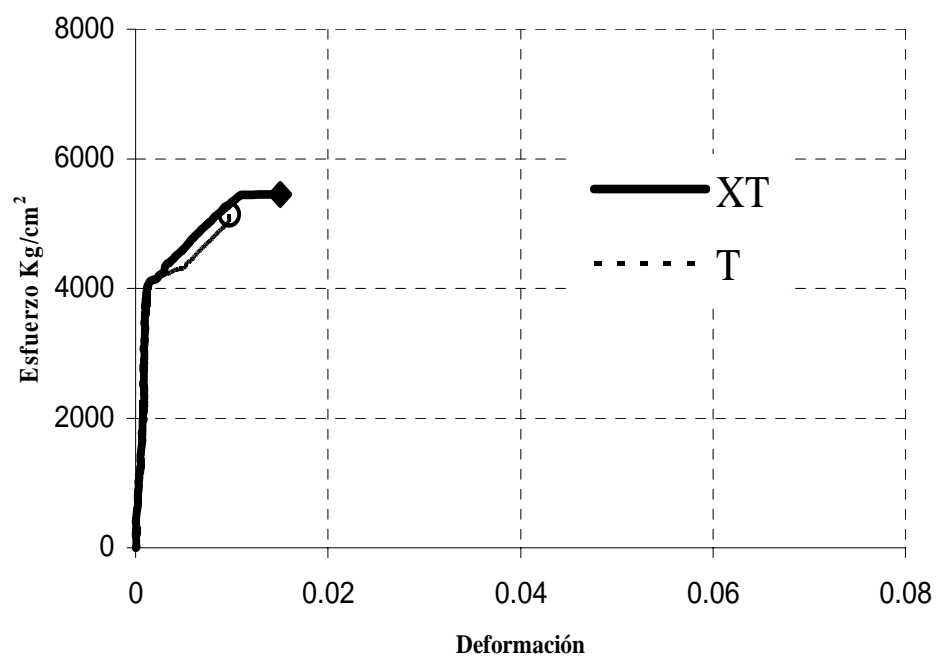

Figura. 15 Diagrama esfuerzo-deformación promedio de barras soldadas No 12. Caso II 


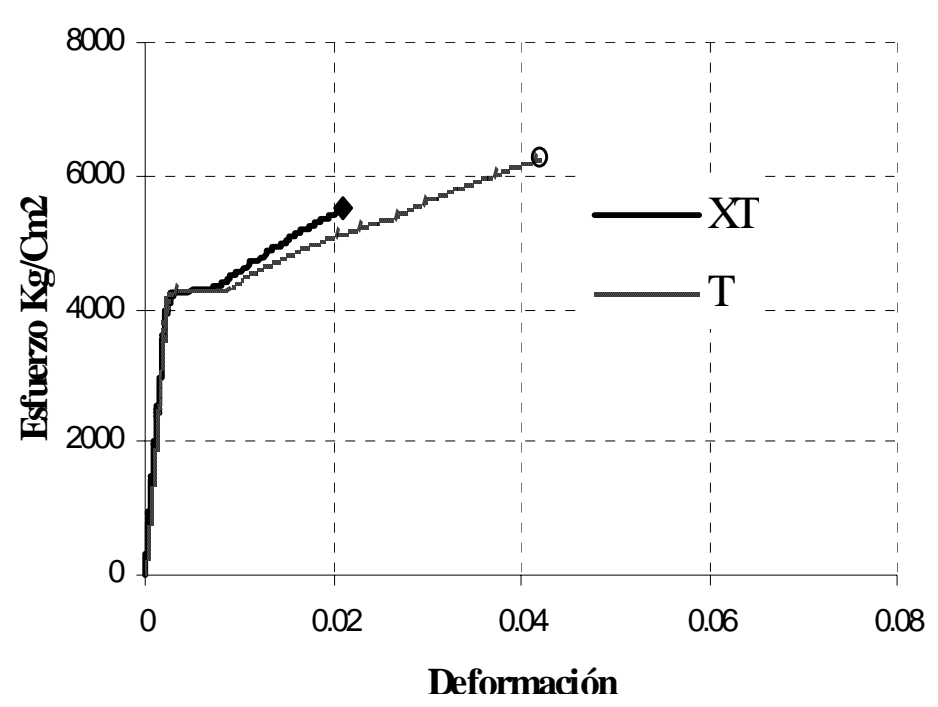

Figura. 16 Diagrama esfuerzo-deformación promedio de barras soldadas No 12. Caso III

La figura 17 permite distinguir dos tipos de falla observados en este estudio en las barras soldadas. La figura 17 (a) muestra el modo de falla de una barra soldada No 10, en la que se observa que la fractura ocurre en la "zona afectada por el calor", la cual es una falla de tipo frágil. La figura 17 (b) muestra el modo de falla de una barra soldada No 8, en la que se observa que la fractura ocurre lejos de la soldadura, con lo que se puede lograr una falla del tipo dúctil. El tipo de falla observado en este estudio en la mayoría de ensayes de barras soldadas fue del tipo frágil, ilustrado en la figura 17 (a), lo que llevó en estos casos a una pobre capacidad de deformación de la barra como se describe en la siguiente parte de este trabajo.

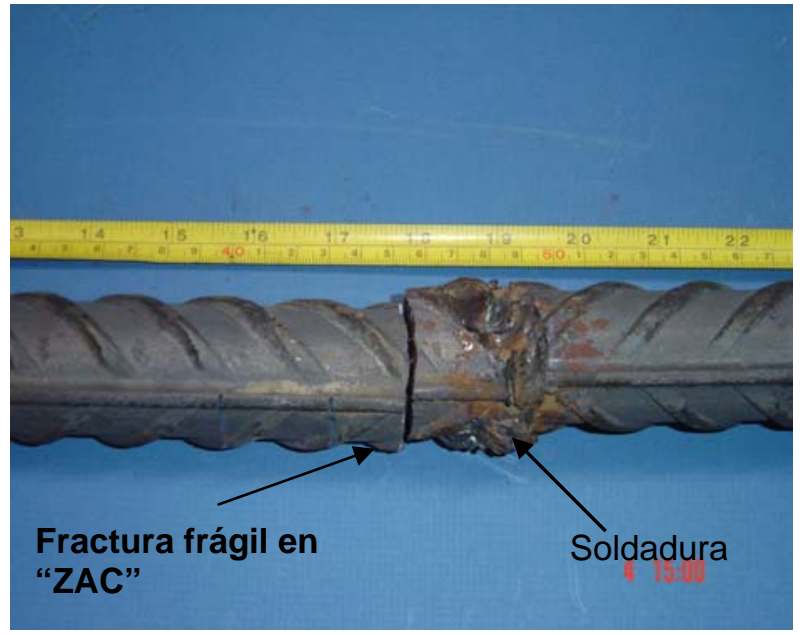

a) Barra No 10

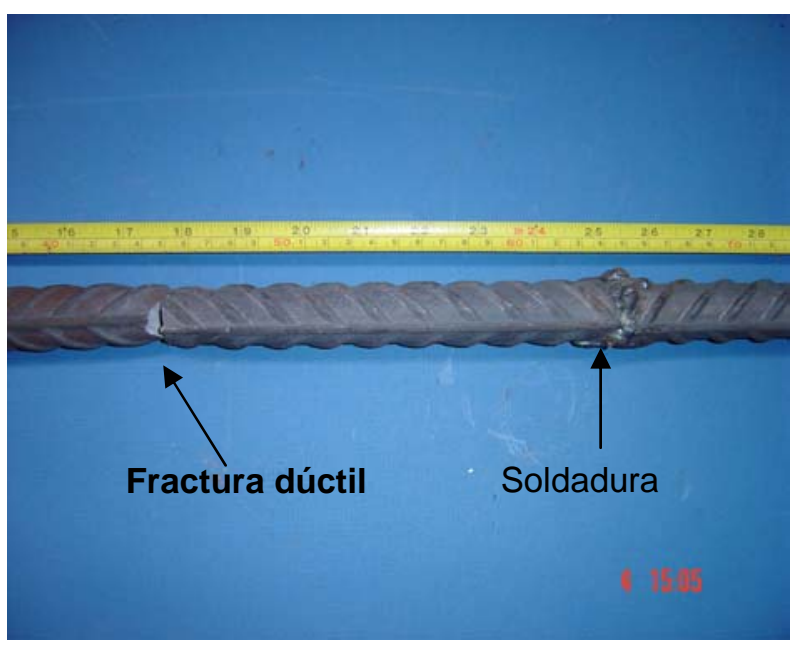

b) Barra No 8

Figura. 17 Barras soldadas con fractura frágil y dúctil 


\section{ANALISIS DE RESULTADOS}

\section{Capacidades resistentes y de deformación requeridas en barras de refuerzo soldadas}

De acuerdo con el Reglamento ANSI/AWS D1.4-98 (AWS, 1998) la resistencia mínima a tensión de las barras soldadas debe ser mayor que 1.25 veces el esfuerzo de fluencia mínimo especificado. Las Normas Técnicas para Diseño y Construcción de Estructuras de Concreto del Distrito Federal (NTCC-DF, 2004) especifica para marcos de ductilidad limitada, con factor de comportamiento sísmico, $Q$, igual a dos, que una unión soldada debe tener una resistencia mínima de 1.25 veces la fuerza de fluencia de tensión de las barras, sin especificar si esta fluencia es la especificada o la medida.

Los autores recomiendan que la resistencia mínima a tensión de una unión soldada debe ser al menos 1.5 veces el esfuerzo de fluencia mínimo especificado. Esta recomendación se basa en resultados de ensayes a tensión de una muestra importante de barras de refuerzo producidas en México, los que indican que en promedio la resistencia a tensión de estas barras fueron mayores que 1.5 veces el esfuerzo de fluencia mínimo especificado (Rodríguez y Botero, 1996). Este requisito es estipulado por la NTCC-DF-2004 para el caso de uniones de barras con dispositivos mecánicos, en secciones críticas donde se espera la formación de articulaciones plásticas en marcos dúctiles. En estas secciones para el caso de marcos dúctiles, la NTCC-DF-2004 no permite el empleo de barras soldadas. Sin embargo, en marcos de ductilidad limitada, esta normativa permite la soldadura de barras en cualquier ubicación. En este caso la NTCC-DF2004 especifica que en una misma sección no se debe soldar más del 33\% del refuerzo, con secciones de unión distantes entre sí no menos de 20 diámetros. Sin embargo, en opinión de los autores esta normativa falla al permitir que no se cumpla estas especificaciones cuando "se garantice una supervisión estricta en la ejecución de las uniones” (sección 5.6.1.3 de la NTCCDF-2004), ya que esta supervisión en realidad es difícil que se de en la práctica. Además, la NTCC-DF-2004 permite que este tipo de marcos de ductilidad limitada se emplee en zonas de alta sismicidad, a diferencia de normativas como la del ACI 318-05 (ACI 318, 2005), que no permite estructuras de ductilidad limitada en zonas de alta sismicidad. Los autores consideran que esta diferencia de criterios se hace más grande y del lado de la inseguridad si en los casos de marcos de ductilidad limitada, diseñados de acuerdo con la NTCC-DF-2004, se permite el empleo de barras soldadas en secciones críticas con una pobre capacidad de deformación.

Es importante enfatizar que ninguna de las normativas aquí mencionadas estipula capacidades de deformación a tensión mínimas en barras soldadas. Los autores consideran que esto es una deficiencia en estas normativas y sugieren que las barras soldadas deben tener una capacidad de deformación a tensión al menos igual a la de las barras que no son soldadas. De acuerdo con la normativa nacional NMX-C-407, el valor mínimo de $\varepsilon_{\text {su }}$ especificado para barras No 8 es 0.08, y para barras No 10 y 12 este valor es 0.07 . Es de interés mencionar que de acuerdo con los resultados de ensayes de las barras testigo, cuyos resultados se muestran en la figura 7 , los valores medidos para $\varepsilon_{\mathrm{su}}$ fueron mayores que los valores mínimos especificados por la normativa nacional. 
Se debe hacer énfasis en la importancia de lograr que las barras de refuerzo soldadas, en estructuras de concreto en zonas sísmicas, tengan suficiente capacidad de deformación, al menos igual a la mínima especificada en la normativa nacional para barras de refuerzo. Esto se puede explicar considerando que en articulaciones plásticas de estructuras en zonas sísmicas, diseñadas de acuerdo con reglamentos con requisitos de ductilidad, el tipo de falla esperado es del tipo de flexión, y en la mayor parte de los casos ocurre con pandeo del refuerzo longitudinal. De acuerdo con algunos autores (Priestley, 2000), para tomar en cuenta de manera aproximada este modo de falla en una evaluación de la capacidad de deformación de una sección crítica de un elemento estructural sometido a acciones sísmicas, se debe considerar que la capacidad a deformación a tensión de una barra de refuerzo es igual a $0.6 \varepsilon_{\text {su }}$. Esta recomendación pone en evidencia la relevancia de lograr en barras de refuerzo soldadas, una capacidad de deformación a tensión mínima que garantice un adecuado comportamiento sísmico de la estructura durante sismos fuertes.

Con base en estas observaciones, y extrapolando el criterio de la normativa NMX-C-407 a las barras soldadas, los autores sugieren que las barras soldadas deben tener al menos las capacidades de deformación mínimas especificadas por esta normativa para barras de refuerzo.

\section{Capacidades resistentes y de deformación observadas en las barras soldadas ensayadas}

La figura 18 muestra capacidades resistentes y de deformación de los seis grupos de barras soldadas, grupos $\mathrm{A}$ al $\mathrm{F}$. Estas capacidades corresponden a los valores promedio de $\varepsilon_{\mathrm{su}} \mathrm{y} \mathrm{F}_{\mathrm{su}} / \mathrm{F}_{\mathrm{y}}$ $\left(F_{y}=4,200 \mathrm{~kg} / \mathrm{cm}^{2}\right)$ de barras soldadas No 8, 10 y 12. En la escala vertical se indica los valores medidos promedio de $\varepsilon_{\text {su }}$ y $F_{\text {su }} / F_{\mathrm{y}}$ y en la escala horizontal se indican los casos considerados por cada variable. La leyenda $\mathrm{XT}$ en la figura 18 significa sin precalentamiento y $\mathrm{T}$ con precalentamiento. La leyenda B1 y B2 en la nota de la figura. 18 se refiere al tipo de bisel empleado, en posición vertical y horizontal, definidos en las figuras 5 y 6, respectivamente. Los seis grupos de barras de refuerzo que intervienen en la figura. 18 se han agrupado en los casos I al III anteriormente descritos, para evaluar el efecto del precalentamiento en las barras. El caso I corresponde a barras con bisel B1 y electrodos E70, el caso II corresponde a barras con el mismo bisel pero con electrodos E90, y el caso III corresponde a barras con bisel B2 y electrodos E90.

El análisis de resultados de la figura 18 indica que el seguir el procedimiento de precalentamiento especificado por la normativa AWS para barras de refuerzo soldadas no llevó a diferencias importantes en las capacidades resistentes y de deformación de las barras No 8, 10 y 12 cuando éstas se unen con diferentes tipos de bisel y de electrodo, excepto en los casos de barras No 10 y No 12 con soldadura de doble bisel y electrodo E90, donde debido al efecto del precalentamiento en estas barras se alcanzaron deformaciones últimas del orden de 0.03 y 0.05 , respectivamente. En estas barras los incrementos de capacidades de deformación respecto al caso de barras sin precalentamiento fueron aproximadamente iguales a $45 \%$ y $95 \%$, respectivamente.

Los resultados de la figura 18 indican que ninguna de las barras soldadas estudiadas en este trabajo cumpliría con el requisito de deformación mínima a tensión especificada por la normativa NMX-C-407 para barras de refuerzo, requisito que en este trabajo se recomienda cumplir para barras soldadas. Solamente las barras No 8, con doble bisel y soldadas con 
electrodos E90 tendrían capacidades de deformación que se acercan al valor mínimo especificado.
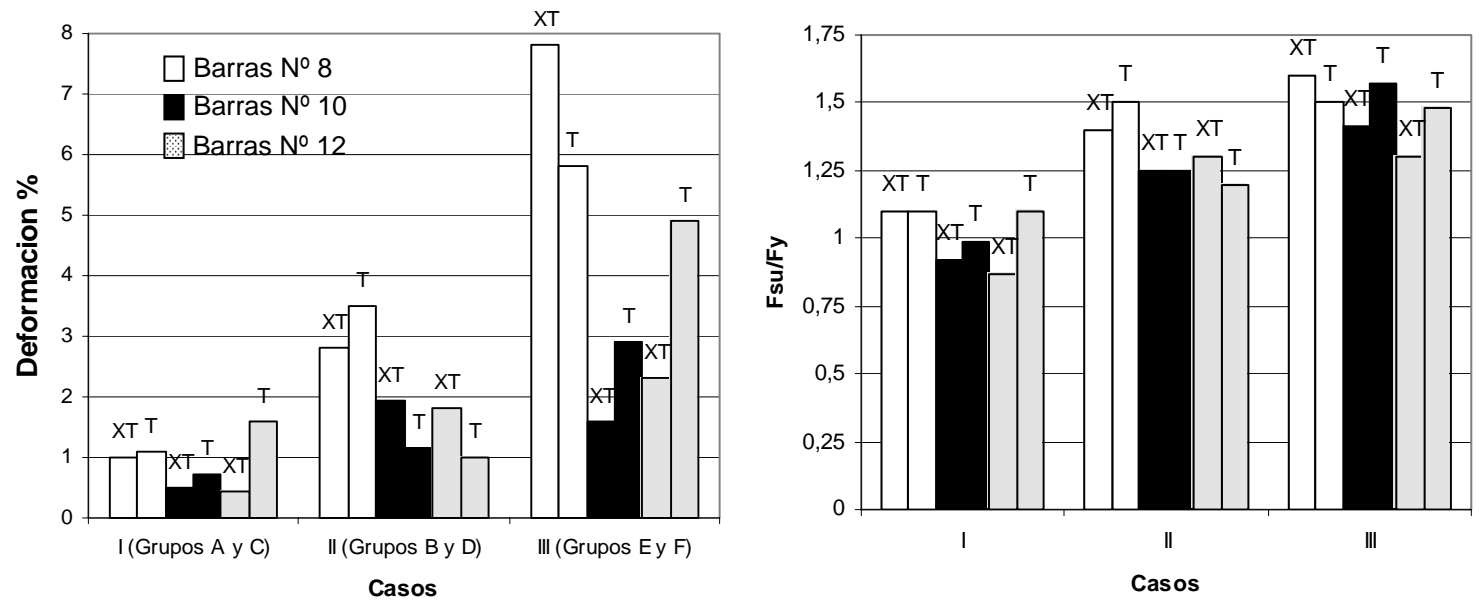

Figura 18. Efecto del precalentamiento del metal base en $\varepsilon_{\mathrm{su}} \mathrm{y} \mathrm{F}_{\mathrm{su}} / \mathrm{F}_{\mathrm{y}}$ en barras soldadas. Nota: Grupos A y C (B1, E70), Grupos B y D (B1, E90), Grupos E y F (B2, E90)

Los resultados de la figura 18 también indican que la práctica común que se sigue en México de soldar con electrodo E70 y en posición vertical (bisel B1), llevaría a valores de la relación $\mathrm{F}_{\mathrm{su}} / \mathrm{F}_{\mathrm{y}}$ inferiores al valor 1.25 recomendado tanto por la AWS como por la NTCC-DF2004. En estos casos se observó que las barras fallaron en la soldadura. Además, en el proceso de soldadura del caso en discusión se observaron problemas como falta de fusión del electrodo, porosidad excesiva e inclusiones de escoria. Estos resultados también indican que si con el tipo de bisel B1 se emplea electrodo E90 sería posible cumplir con el mínimo recomendado para la relación $\mathrm{F}_{\mathrm{su}} / \mathrm{F}_{\mathrm{y}}$ por la AWS y la NTCC-DF-2004; sin embargo, no se alcanzaría el valor de 1.5 para esta relación como se ha recomendado en este estudio. Sólo si se emplea doble bisel (bisel B2) con electrodos E90 se estaría cerca o se superaría el valor 1.5 para la relación $\mathrm{F}_{\mathrm{su}} / \mathrm{F}_{\mathrm{y}}$.

Para evaluar el efecto del tipo de bisel en las capacidades resistentes y de deformación de barras soldadas, se comparan resultados de los grupos B, D, E y F. Estos resultados se muestran en la figura 19, correspondientes a barras soldadas con electrodo E90 y diferentes tipos de bisel, B1 y B2. En esta figura se tienen resultados de los casos IV (grupos B y E, sin precalentamiento), y el caso $\mathrm{V}$ (grupos $\mathrm{D}$ y F, con precalentamiento). Como se aprecia en la figura 19, el efecto del tipo de bisel es relevante para barras del diámetro No 8, y en barras No 10 y 12 con precalentamiento. Sin embargo, como se ha mencionado, ninguna de estas barras alcanza la capacidad de deformación mínima recomendada en este estudio. Como se aprecia en la figura 19, en prácticamente todos los casos analizados en esta figura se alcanzaría el valor mínimo para la relación $\mathrm{F}_{\mathrm{su}} / \mathrm{F}_{\mathrm{y}}$ recomendado por la AWS y la NTCC-DF-2004; sin embargo, en barras soldadas No 10 y 12, sólo empleando doble bisel, electrodos E90 y con precalentamiento, se estaría cerca al valor 1.5 recomendado en este estudio para la relación $F_{\text {su }} / F_{y}$. 

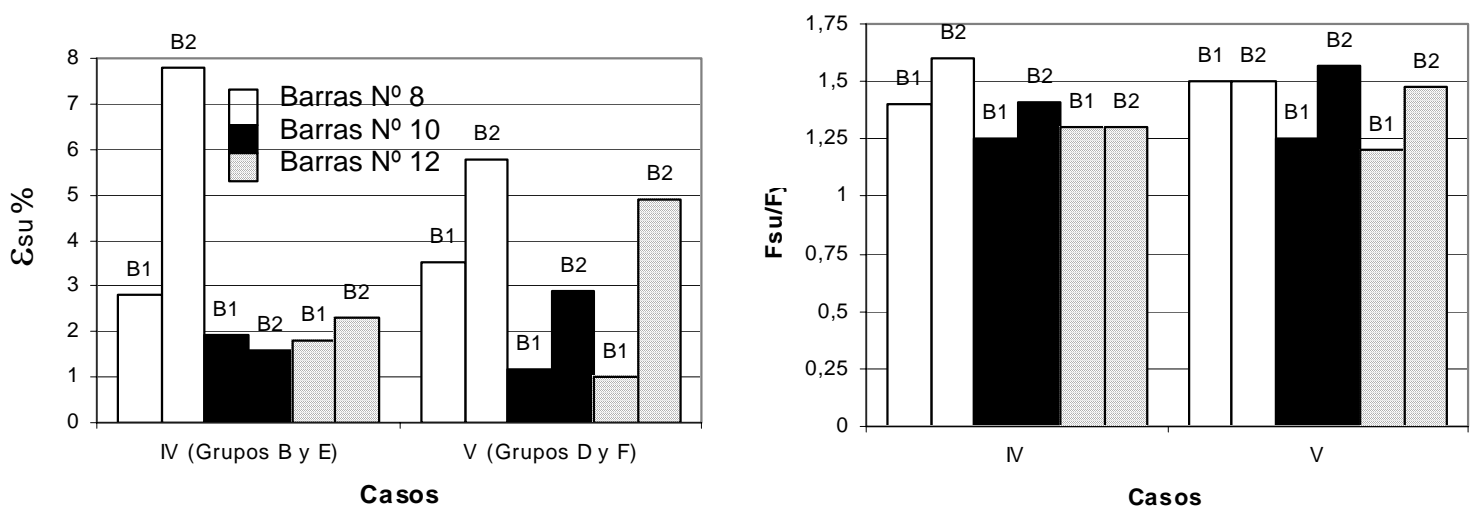

Figura 19. Efecto del tipo de bisel en $\varepsilon_{\mathrm{su}} \mathrm{y} \mathrm{F}_{\mathrm{su}} / \mathrm{F}_{\mathrm{y}}$ en barras soldadas. Nota: Grupos B y E (XT, E90), Grupos D y F (T, E90)

Finalmente para evaluar el efecto del tipo de electrodo en barras soldadas se comparan resultados de los grupos A al D. Estos resultados se muestran en la figura 20, correspondientes a barras soldadas con los tipos de electrodos E70 y E90 y con bisel B1. En esta figura se tienen resultados de los casos VI (grupos Ay B, sin precalentamiento) y VII (grupos C y D, con precalentamiento). Como se aprecia en la figura 20, en general se observa que el empleo de electrodos E90, comparado con el empleo de electrodo E70, lleva a aumentos de la capacidades resistente a tensión y de deformación. Sin embargo, se observa que en ninguno de estos casos se alcanza la deformación mínima a tensión recomendada en este estudio. En lo referente a capacidad resistente, como se ha mencionado con el electrodo E70 no se alcanza la capacidad resistente mínima a tensión requerida por la AWS y la NTCC-DF-2004, necesitándose electrodos E90 para lograr esta capacidad, aún cuando en los casos analizados no se supera el valor 1.5 recomendado en este estudio para la relación $\mathrm{F}_{\mathrm{su}} / \mathrm{F}_{\mathrm{y}}$.
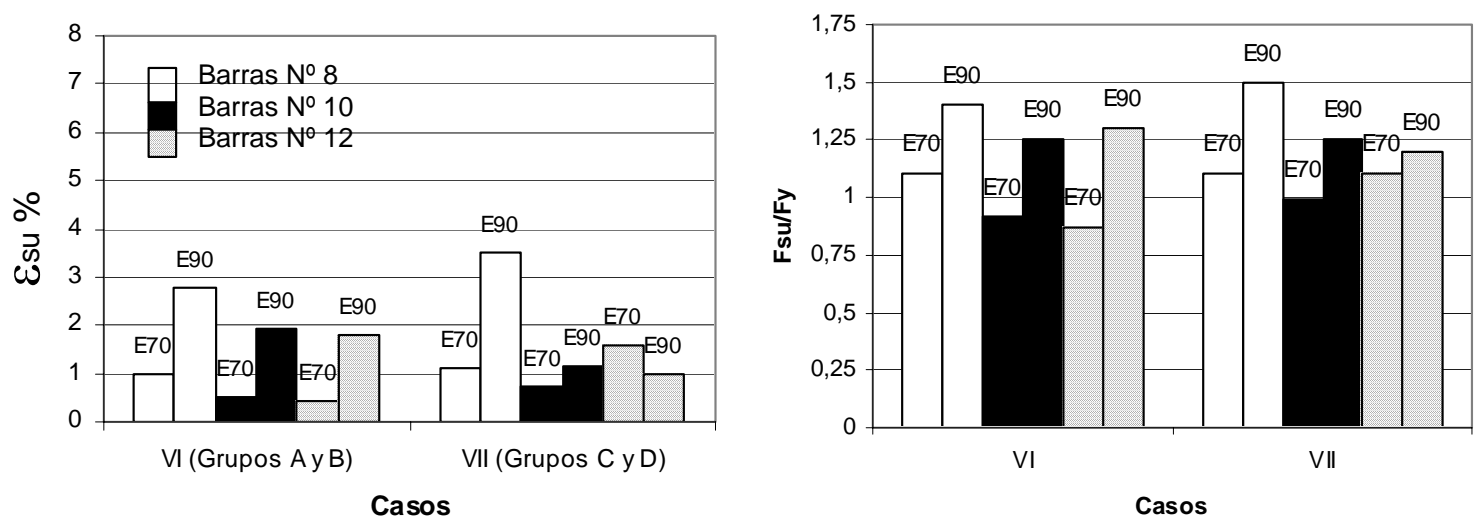

Figura 20. Efecto del tipo de electrodo en $\varepsilon_{\mathrm{su}} \mathrm{y} \mathrm{F}_{\mathrm{su}} / \mathrm{F}_{\mathrm{y}}$ en barras soldadas. Nota: Grupos A y B (XT, B1), Grupos C y D (T, B1) 
Los ensayes de barras soldadas aquí descritos son del tipo monótono, es decir no fueron del tipo cíclico reversible, típico de acciones sísmicas; sin embargo, sus resultados se pueden extrapolar a los del tipo cíclico si se considera que modelos analíticos del comportamiento cíclico de barras de refuerzo se basan en el empleo de reglas de cargas y descargas en las curvas esfuerzo-deformación que emplean características de las curvas de tipo monótono.

\section{SOLDADURA DE BARRAS DE REFUERZO EN EDIFICIOS DE CONCRETO REFORZADO EN MEXICO}

El sistema estructural sismorresistente más empleado para la construcción de edificios de concreto reforzado en México es el sistema a base de marcos. Tanto en los casos de edificios con elementos estructurales colados en sitio, como en los construidos con elementos prefabricados, en general las barras de refuerzo de diámetro mayores que el No 8 se conectan empleando soldadura. En el caso de estructuras prefabricadas, el problema de la soldadura de barras de refuerzo puede ser mas relevante que en el caso de estructuras de concreto colado en sitio, ya que es común que las conexiones entre elementos prefabricados se resuelvan mediante la soldadura de barras de refuerzo, lo que ante acciones sísmicas podría llevar en marcos de concreto reforzado prefabricados a una mayor vulnerabilidad que en los colados en sitio.

Para el estudio de este problema se han seleccionado dos casos de edificios prefabricados en la ciudad de México. La figura 21 muestra una conexión trabe-columna entre elementos prefabricados de concreto para un edificio a base de marcos de 15 niveles, construido en el año 2001. Como se aprecia en la figura 21, la continuidad del refuerzo del lecho superior se intentó resolver mediante soldadura de dos placas de acero con tramos de barras de refuerzo del No 10. Una de estas placas está embebida en la ménsula de la trabe y la otra placa está embebida en la ménsula de la columna. Una solución semejante se empleó para intentar lograr la continuidad del refuerzo del lecho inferior. Como se ha mostrado en este estudio, la soldadura en barras de refuerzo produce en éstas la denominada “zona afectada por el calor”, la que produce un tipo de falla frágil cuando la barra soldada incursiona en tensión en el intervalo inelástico, lo que puede ocurrir cuando una estructura responde ante sismos fuertes. Esto sugiere que cuando el edificio que aquí se comenta tenga incursiones inelásticas en un sismo fuerte, la referida conexión no podría ser capaz de tener la capacidad de deformación en tensión requerida para disipar la energía introducida por el sismo, provocando la falla de la sección del elemento estructural y por tanto afectando seriamente la seguridad estructural del edificio.

La figura 22 muestra una conexión trabe-columna y una conexión columna-columna, empleadas en un edificio prefabricado de concreto de doce niveles, construido en el año 2005 en la ciudad de México. En estas conexiones se empleó barras de refuerzo de diámetro No 12, las que fueron soldadas con electrodos E70 y sin precalentamiento. La soldadura de barras de la conexión trabe-columna se hizo en posición horizontal, con un ángulo de respaldo. La soldadura en la conexión columna-columna se hizo en posición vertical, con un bisel a $45^{\circ}$, también con ángulo de respaldo. Con base en los resultados encontrados en este estudio, en las barras soldadas empleadas en las conexiones aquí descritas es de esperar que la capacidad de deformación en tensión de estas barras no supere el valor 0.02. Este valor es bastante menor que 
el valor de 0.07 recomendado en este estudio para barras de diámetro No 12, con el fin de lograr un comportamiento sísmico adecuado de la sección crítica durante un sismo fuerte.

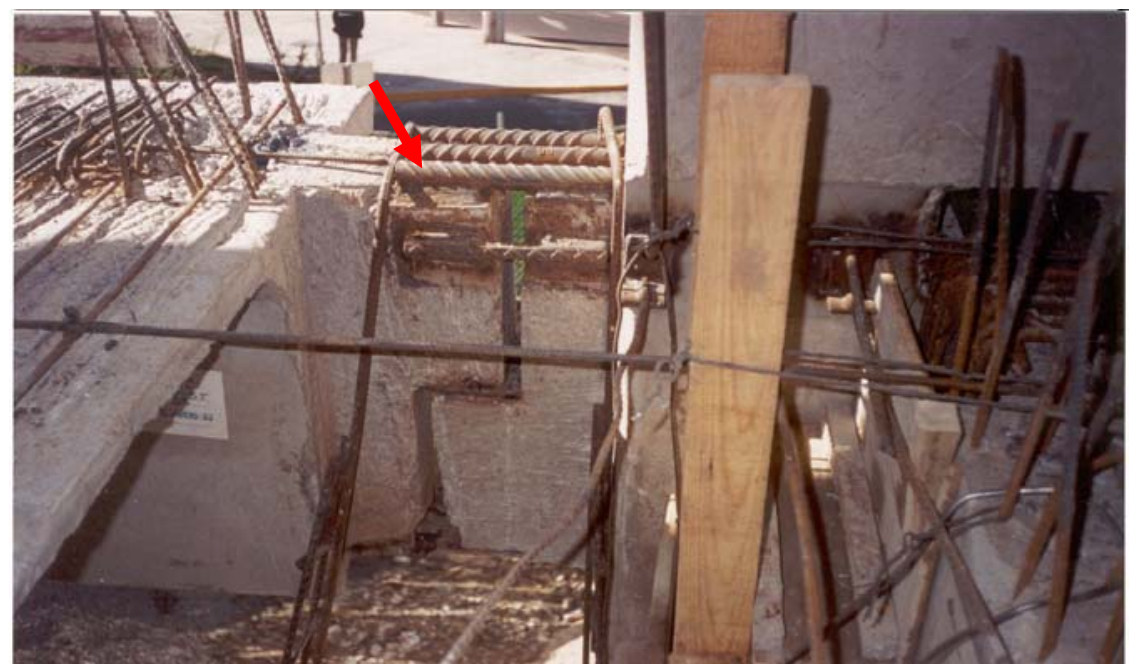

Figura 21. Conexión trabe-columna en un edificio prefabricado de concreto en México que emplea soldadura de barras de refuerzo en placas.

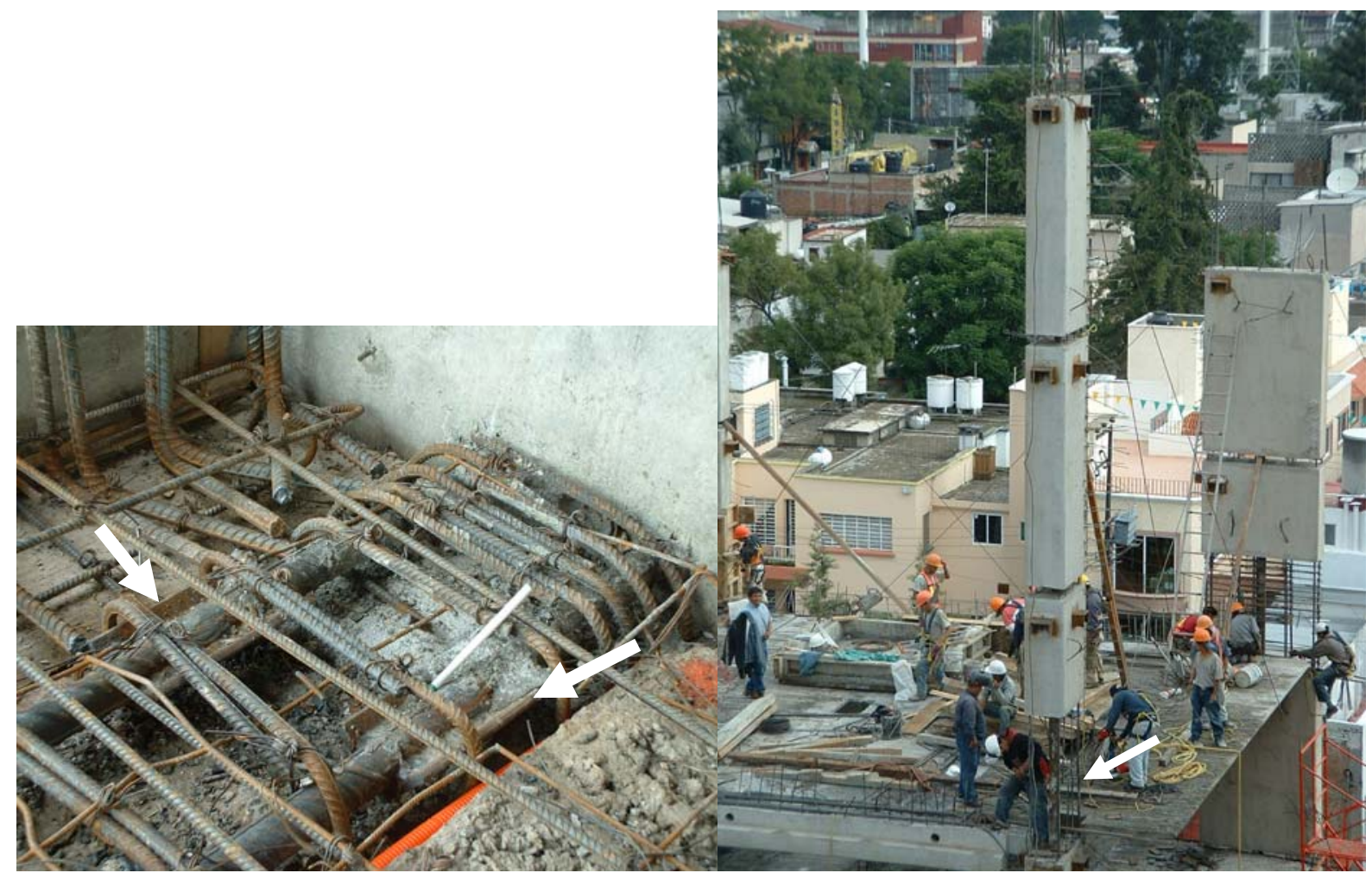

a) Conexión trabe-columna

b) Conexión columna-columna

Figura 22. Conexiones en un edificio prefabricado de concreto en México que emplean soldadura de barras de refuerzo. 
Los casos de soldadura de barras de refuerzo en conexiones de elementos prefabricados, como en los edificios aquí analizados, no son aislados. Los autores sugieren un cambio urgente de estas prácticas constructivas de edificios prefabricados en México, en el que se evite la soldadura de barras de refuerzo en estructuras en zonas sísmicas de nuestro país.

\section{CONCLUSIONES}

En esta investigación se llevó a cabo ensayes en tensión de 54 barras de refuerzo, de diámetros No 8, 10 y 12, las que fueron soldadas a tope directo. Se evaluó el efecto de tres variables importantes en el proceso de soldadura, el tipo de electrodo (E-7018 y E-9018), el tipo de bisel y el efecto del precalentamiento. De este estudio se obtuvieron las siguientes conclusiones:

1) Se recomienda no usar electrodos E7018 para soldar barras de refuerzo, debido a que los resultados de los ensayes mostraron que para este caso la capacidad de deformación última no llegó a 0.02, valor bastante menor que el recomendado en este estudio como capacidad mínima de deformación. Además los valores medidos de la relación $\mathrm{F}_{\mathrm{su}} / \mathrm{F}_{\mathrm{y}}$ fueron menores que el valor mínimo de 1.25 recomendado para esta relación tanto por la AWS como por la NTCC-DF-2004.

2) El empleo del precalentamiento y electrodos E9018 en barras soldadas en posición vertical llevó, respecto al caso anterior con electrodos E70, a ligeras mejoras en la capacidad de deformación y en los valores de la relación $\mathrm{F}_{\mathrm{su}} / \mathrm{F}_{\mathrm{y}}$. Sin embargo, las capacidades de deformación medidas fueron menores que la mínima recomendada en este estudio, y para barras de diámetros No $10(31.8 \mathrm{~mm})$ y No $12(38.1 \mathrm{~mm})$, la relación $\mathrm{F}_{\mathrm{su}} / \mathrm{F}_{\mathrm{y}}$ apenas alcanzó el valor 1.25 recomendado por la AWS y la NTCC-DF-2004, y fue menor que el valor recomendado en este estudio.

3) En todas las barras soldadas con electrodos E9018, excepto las barras No 8 (25.4 mm) soldadas en posición horizontal con doble bisel, la falla ocurrió de modo frágil en la soldadura y en la "zona afectada por el calor". El precalentamiento de $150^{\circ} \mathrm{C}$ recomendado por la AWS no fue suficiente para evitar en estos casos la falla de las barras soldadas en la "zona afectada por el calor".

4) Con base en los resultados de esta investigación aquí comentados, en estructuras de concreto reforzado en zonas sísmicas de nuestro país se recomienda no soldar barras de refuerzo con características de la normativa NMX-C-407 con diámetros mayores que el diámetro $\mathrm{N}^{\circ} 8(25.4 \mathrm{~mm})$. Los resultados encontrados en este estudio muestran que sólo las barras de refuerzo No 8 soldadas con doble bisel y electrodos E90 podrían alcanzar valores recomendados de capacidades resistentes y de deformación de barras de refuerzo en elementos de concreto reforzado que son sometidos a acciones sísmicas. 
5) Los casos de barras soldadas estudiadas en esta investigación que presentaron un pobre comportamiento sísmico, como escasa capacidad de deformación y baja relación $\mathrm{F}_{\mathrm{su}} / \mathrm{F}_{\mathrm{y}}$, son representativas de prácticas usuales de construcción en nuestro país. Por este motivo, en un evento sísmico, como el correspondiente al de diseño, es de esperar un tipo de falla frágil en secciones críticas de elementos de concreto con barras de refuerzo con estos tipos de soldaduras, con un comportamiento indeseable desde el punto de vista del diseño sismorresistente.

6) Debido a las limitaciones aquí descritas de pobres características de soldabilidad de las barras de refuerzo de acero que se usan en nuestro país, con características de la normativa NMX-C-407 (ASTM A615), se recomienda que este tipo de acero no se emplee en zonas sísmicas de nuestro país. Por las buenas características de soldabilidad de barras de refuerzo con aceros de baja aleación, especificados por la normativa Mexicana NMX-B-457 (ASTM A706), se recomienda empezar la producción en México de este tipo de refuerzo para ser empleado en construcciones en zonas sísmicas de nuestro país, en lugar de barras de acero tipo NMX-C-407 (ASTM A615).

7) Una alternativa a la soldadura en barras de refuerzo que se podría explorar es el empleo de conectores mecánicos para barras de refuerzo, siempre y cuando se verifique que estos conectores tengan las capacidades resistentes y de deformación que se recomiendan en este trabajo. Un estudio de este tipo se lleva a cabo actualmente en el Instituto de Ingeniería de la UNAM.

\section{AGRADECIMIENTOS}

Este estudio fue parte de un proyecto de investigación (No 5520) llevado a cabo en el Instituto de Ingeniería de la UNAM (II-UNAM). Los fondos para esta investigación provinieron de un premio que en el año 2003 otorgó la CANACERO al II-UNAM por una investigación previa del Doctor Mario E. Rodríguez. Se agradece la colaboración en esta investigación de personal del IIUNAM, Dr Carlos Aire, Ing Fabián De la Serna y Sr Raymundo Mondragón. Se agradece al Ing Francisco García Jarque, de García Jarque Ingenieros, sus comentarios al manuscrito, que permitieron mejorarlo.

\section{REFERENCIAS}

ACI Committee 318 (ACI 318, 2005), "Building Code Requirements for Reinforced Concrete (ACI 318-05)”. American Concrete Institute, Farmington Hills, MI.

American Society for Metals (ASM, 1990, 1992), ASM Handbook, Vol 1 y 6, $10^{a}$ Edición.

American Welding Society (AWS, 1998). "Structural Welding Code-Reinforcing Steel” NSI/AWS D1.4-98.

American Welding Society (AWS, 1968 y 1987), “Welding Handbook” 6ª y 8 Edición. 
American Society for Testing and Materials (1992). "Low-Alloy Steel Deformed Bars for Concrete Reinforcement”, ASTM A706/A 706M-92b.

American Society for Testing and Materials (1992). "Deformed and Plain Billet-Steel Bars for Concrete Reinforcement”, ASTM A615/A 615M-92b.

Dirección General de Normas (1988), "Procedimiento de Soldadura Estructural Acero de Refuerzo” Norma Mexicana NMX-H-121-1988, Secretaría de Comercio y Fomento Industrial.

Dirección General de Normas (2001), "Varilla Corrugada de Acero Proveniente de Lingote y Palanquilla para Refuerzo de Concreto" Norma Mexicana NMX-C-407-ONNCCE-2001, Organismo Nacional de Normalización y Certificación de la Construcción y Edificación, S. C.

Dirección General de Normas (1988). "Varillas Corrugadas de Acero de Baja Aleación Procedentes de Lingote o Palanquilla para Refuerzo de Concreto". Norma Mexicana NMX-B457-1988.

Gaceta Oficial del Distrito Federal (2004), "Normas Técnicas Complementarias para Diseño y Construcción de Estructuras de Concreto" Reglamento de Construcciones del Distrito Federal. México DF.

Gustafson, D P y A L Felder, (1991), “Questions and Answers on ASTM A 706 Reinforcing Bars”, Concrete International, ACI, Vol 13, No 7, pp 54-57.

Gustafson D P, (2007), "Revisiting Low-Alloy Steel Reinforcing Bars”, Concrete International, ACI, Enero, 55-59.

Priestley N J M, (2000), “Performance Based Seismic Design”, Proc. 12 World Conference on Earthquake Engineering, Auckland, Nueva Zelandia, paper No 2831,

Rodríguez M y J C Botero, (1996), “Aspectos del comportamiento sísmico de estructuras de concreto reforzado considerando las propiedades mecánicas de aceros de refuerzo producidos en México”. Publicación Series del Instituto de Ingeniería, UNAM, No 575, enero 1996.

Rodríguez M y J Rodríguez, (2007), "Soldadura de barras de refuerzo para estructuras de concreto reforzado en zonas sísmicas”. SID 653, Serie Investigación y Desarrollo del Instituto de Ingeniería, UNAM. 